\title{
Modelling dose rate to single grains of quartz in well-sorted sand samples: The dispersion arising from the presence of potassium feldspars and implications for single grain OSL dating
}

Guerin, Guillaume; Jain, Mayank; Thomsen, Kristina Jørkov; Murray, Andrew Sean; Mercier, Norbert

Published in:

Quaternary Geochronology

Link to article, DOI:

10.1016/j.quageo.2014.12.006

Publication date:

2015

Document Version

Peer reviewed version

Link back to DTU Orbit

Citation (APA):

Guerin, G., Jain, M., Thomsen, K. J., Murray, A. S., \& Mercier, N. (2015). Modelling dose rate to single grains of quartz in well-sorted sand samples: The dispersion arising from the presence of potassium feldspars and implications for single grain OSL dating. Quaternary Geochronology, 27, 52-65.

https://doi.org/10.1016/j.quageo.2014.12.006

\section{General rights}

Copyright and moral rights for the publications made accessible in the public portal are retained by the authors and/or other copyright owners and it is a condition of accessing publications that users recognise and abide by the legal requirements associated with these rights.

- Users may download and print one copy of any publication from the public portal for the purpose of private study or research.

- You may not further distribute the material or use it for any profit-making activity or commercial gain

- You may freely distribute the URL identifying the publication in the public portal 
1 Modelling dose rate to single grains of quartz in well-sorted sand samples: the dispersion arising from

2 the presence of potassium feldspars and implications for single grain OSL dating

3 Guillaume Guérin ${ }^{1,2}$, Mayank Jain ${ }^{1}$, Kristina Thomsen ${ }^{1}$, Andrew Murray ${ }^{3}$, Norbert Mercier ${ }^{2}$.

$4{ }^{1}$ Center for Nuclear Technologies, Technical University of Denmark, DTU Risø Campus, DK-4000 Roskilde, 5 Denmark.

$6{ }^{2}$ Institut de Recherche sur les Archéomatériaux, UMR 5060 CNRS - Université de Bordeaux, Centre de

7 Recherche en Physique Appliquée à l'Archéologie (CRP2A), Maison de l'archéologie, 33607 Pessac cedex.

$8{ }^{3}$ Nordic Laboratory for Luminescence Dating, Department of Geoscience, Aarhus University, DTUNutech, 9 Risø Campus, DK-4000 Roskilde, Denmark.

10

11 Corresponding author: G. Guérin (guillaume.guerin@u-bordeaux-montaigne.fr)

12

13 
14

\section{Abstract}

Single grain OSL has become a widely used approach in Quaternary geochronology. However, the origins of $D_{e}$ distributions and the sources of variation in individual dose estimates are still poorly understood. The amount of scatter in these distributions on top of the known uncertainties in measurement and analysis is defined by overdispersion and this quantity is generally used for weighting individual $D_{e}$ values to calculate a central equivalent dose. In this study, we address the nature and amount of different sources of dispersion in quartz single grain $D_{e}$ estimates, by (i) using appropriate statistical tools to characterize $D_{e}$ populations and (ii) modelling, with a specifically designed GEANT4 code, dose rate distributions arising from the presence of potassium feldspar grains in well-sorted sands. The model uses Monte Carlo simulations of beta emissions and interactions in a random close packing of quartz and feldspar spheres representing a sand sample. Based on the simulation results, we explain the discrepancy between intrinsic and natural overdispersion values in a well-bleached sample, thus validating the model. The three parameters having the most influence on dispersion in dose rate distributions, and modelled in this study, appear to be grain size, potassium content and total dose rate.

Finally an analysis of measurement uncertainties and other sources of variations in equivalent dose estimates leads us to conclude that all age models (both logged and unlogged) which include an overdispersion value to weight individual $D_{e}$ values rely mainly on unknown parameters; this ignorance may lead to an inadvertent bias in $D_{e}$ estimates. Assuming counting statistics make a small contribution to dispersion (as is often the case), we suggest that in some cases it is most appropriate to use unweighted averages of equivalent doses when dividing by commonly measured average dose rates. 


\section{Introduction.}

Quartz Optically Stimulated Luminescence (OSL) has become a widely used tool for establishing

39 the chronology of sediment burial. The Single Aliquot Regenerative protocol (SAR: Murray and Wintle, 2000; 2003) allows the determination of individual equivalent dose estimates $\left(D_{e}\right)$ from aliquots of

41 arbitrary number of grains, including individual grains. Equivalent dose distributions derived from single-

42 grain measurements are usually significantly dispersed, requiring some statistical treatment for their

43 analysis; the choice of this statistical treatment can have a significant effect on the accuracy of the

44 resulting OSL ages. For instance, post-depositional mixing of sediments (e.g., Tribolo et al., 2010) and/or

45 insufficient resetting of the OSL signal before deposition (e.g., Jain et al., 2004; Olley et al., 2004) may

46 lead to dose distributions where the central value is not representative of the sediment burial event. In

47 single-grain equivalent dose analysis, the key concept of overdispersion (OD) is defined as the dispersion

48 of results that cannot be explained by 'within aliquot errors', i.e. the measured or otherwise known

49 uncertainties assigned to individual equivalent dose estimates (see Galbraith et al., 1999, for an

50 introduction and discussion on its significance in OSL dating; see also Galbraith and Roberts, 2012).

51 Statistical models have been proposed to identify the $D_{e}$ representative of the target event. For example

52 the Minimum Age Model (MAM, Galbraith et al., 1999), the IEU (Thomsen et al., 2007; Jain et al., 2004)

53 and the leading edge model (Lepper, 2001), have been suggested as tools to resolve the best-bleached

54 component, and the Finite Mixture Model (FMM, Galbraith and Green, 1990; Roberts et al., 2000) has

55 been suggested to identify individual dose components present in a mixture. These models require the

56 input of an estimate of OD appropriate to the sample had it been well bleached; this can be either taken

57 as a value presumed to be typical of well-bleached samples in general (i.e. $<20 \%$, Jacobs et al., 2008a) or 
experimentally determined from well-bleached samples with similar characteristics to those of the

59 sample under investigation (Thomsen et al., 2007).

However, little is known about the nature and source(s) of overdispersion in single grain $D_{e}$ distributions. Thomsen et al. (2012) have demonstrated that overdispersion is dependent on dose in well-bleached samples irradiated with a known gamma dose; in two samples they found the overdispersion increased as the given dose increased. In naturally irradiated samples, different beta dose rates to different grains in sedimentary media are also expected to contribute to overdispersion in $D_{e}$ values (e.g., Mayya et al., 2006; Cunningham et al., 2012). These different dose rates arise because the range of beta particles is comparable to the size of sand grains, and to the inter-granular distance. In

67 particular, the presence of hotspots - such as potassium feldspar grains, which generally represent an important source of dose rates in sands - generates skewed, wide dose rate distributions (Mayya et al., 2006; see also Brennan, 2006, for a discussion on the effect of hotspots on alpha dose rate distributions). Mayya et al. (2006) simulated beta dose rate distributions from individual potassium-rich feldspar grains to single $200 \mu \mathrm{m}$ grains of quartz, and they showed that the dispersion in beta dose rates

72 from potassium increases as the average potassium content (i.e. the number of feldspar grains) is

73 decreased. Nathan et al. (2003) compared experimental and simulation results, using the Monte Carlo radiation transport code MCNP transport code, for different cases of heterogeneity in sedimentary environments. Despite weak agreement between experimental and numerical datasets, they showed that beta dose rate heterogeneity (either in the form of cold or hotspots) can influence single grain $D_{e}$

77 distributions. Cunningham et al. (2012) used MCNP to simulate dose rate distributions induced by $\mathrm{NaOH}$ 78 grains containing artificially produced, short-lived ${ }^{24} \mathrm{Na}$ to mimic the effect of potassium feldspar grains.

79 They were able to reproduce the shape of experimentally determined dose rate distributions, which can 80 be fitted with log-normal distributions, but did not manage to get quantitative agreement between 81 modelled and experimental data. Nevertheless, it is now clear that the presence of radioactive hotspots 
82 induces positively skewed distributions of dose rates; conversely, the presence of coldspots such as

83 calcareous blocks in 'lumpy environments' leads to negatively skewed distributions (see Brennan et al.,

84 1997, for a study of gamma dose rates). These distributions are in contrast to those postulated by Jacobs

85 et al. (2008b) who suggested that coldspots were the explanation for the two discrete modes in their

86 dose distributions; both in view of the experimental and modelling results above, this seems unlikely

87 (see also Guérin et al., 2013).

Despite this general understanding of the effect of hotspots in governing dose distributions, very few studies have compared experimental equivalent dose with simulated dose rate distributions.

Recently Chauhan and Singhvi (2011) compared measured equivalent dose with modelled dose rate distributions, to assess whether the measured dispersion in $D_{e}$ values from multi-grain aliquots could be explained solely by dose rate distributions, or if an extra-source of dispersion such as poor bleaching was needed to explain the scatter in $D_{e}$ measurements. However, this study was not based on single grain $D_{e}$ measurements and it is not clear how many sensitive grains were present per aliquot. Moreover, the dispersion in $D_{e}$ values was taken as the standard deviation of individual estimates, and it did not account for the uncertainties on the individual $D_{e}$ values. In the absence of the knowledge of the effect of these uncertainties, it is difficult to interpret these results quantitatively.

\section{Background}

The purpose of this study is to study beta dose rate distributions from potassium feldspar grains to single grains of quartz in sand using the radiation transport toolkit GEANT4 (Agostinelli et al., 2003). In particular, parameters influencing these dose rate distributions are identified and the model has been tested on a well-bleached, well characterised sand sample. A statistical analysis of $D_{e}$ distributions from both natural and gamma dosed fractions of the sample are provided, and consequences regarding the use of various published age models is discussed. 
106 apply weighting factors to calculate representative equivalent doses. Moreover, most $D_{e}$ distributions

107 reported in the literature exhibit overdispersion. In the most commonly used logged age models (such

108 as for example the Central Age Model and the Minimum Age Model; Galbraith et al., 1999), the same

109 relative OD (in \%) is added in quadrature to individual relative $D_{e}$ uncertainties, assuming multiplicative

110 error properties (i.e. absolute uncertainties proportional to doses); the weighted average of logged $D_{e}$

111 values (geometric mean) corresponds to the central dose. Conversely, in unlogged age models the same

112 absolute OD (in Gy) is added in quadrature to individual absolute $\mathrm{D}_{\mathrm{e}}$ uncertainties, assuming additive

113 error properties (i.e. constant absolute errors); the weighted average of $D_{e}$ values (arithmetic mean)

114 corresponds to the central dose. In both cases the OD parameter is added in quadrature to each dose

115 estimate in the weighted mean calculation of $D_{e}$. The choice between logged or unlogged models

116 depends on the shape of measured $D_{e}$ distributions: multiplicative error properties lead to lognormal

117 distributions (and to the choice of logged age models), whereas additive error properties lead to normal

118 distributions (and to the choice of unlogged age models; for a discussion on this point, see Arnold et al.,

119 2009).

Thomsen et al. (2012) tried to determine whether dose distributions from uniformly gamma

121 irradiated samples were normal or lognormal: they studied $D_{e}$ distributions of samples bleached in a

122 solar simulator and then delivered a homogeneous well-known gamma dose, to study the nature of

123 intrinsic overdispersion. They concluded that both logged and unlogged models provided reasonable,

124 but not perfect fits to their $D_{e}$ distributions; in particular, they found no evidence for multiplicative error

125 properties in equivalent dose measurements that could justify using logged age models.

For this study, a sand sample from a beach-ridge from Skagen (Denmark; see Buylaert et al.,

127 2006; Nielsen et al., 2006; Guérin et al., 2012) was chosen for two reasons: firstly, because its OSL 
properties satisfy the general criteria for acceptability of the SAR protocol (fast component, recycling,

129 recuperation, dose recovery etc.) and in this area, the average OSL ages determined with large multi-

130 grain aliquots of quartz are, for a number of sediment samples $(n=20)$, in good agreement with

131 radiocarbon data (Nielsen et al., 2006); secondly, the beta dose rate from potassium contributes a

132 significant fraction ( $50 \%$ ) of the total dose rate to quartz; hence it is likely that, if dose rate distributions

133 are affected by potassium and have implications regarding single-grain $D_{e}$ populations, such an effect

134 will be observed in this sample. It thus is a good candidate to (i) model beta dose rate distributions from

135 potassium and (ii) experimentally characterise the implications of such modelling for analysis of

136 equivalent dose distributions. As a result, the effect of potassium feldspar grains on the dispersion of $D_{e}$

137 measurements from the natural distribution is presumed to be significant. Following Buylaert et al.

138 (2006), this sample will be referred to as 'the inter-comparison sample'.

\section{Samples, material and methods}

\subsection{Sample preparation and characterization}

\section{Gamma spectrometry}

Sediment was homogenised by crushing and sealed in a plastic box containing $\sim 10 \mathrm{~g}$ of material.

This sealed sample was then stored for at least three weeks to ensure radon build-up, before

144 measurement using high resolution, low background gamma spectrometry, at the IRAMAT-CRP2A in

145 Bordeaux. The potassium, uranium and thorium contents are given in Table 1. The corresponding dose

146 rates have been calculated using dose rate conversion factors from Guérin et al. (2011) and using grain-

147 size attenuation factors from Guérin et al. (2012). The accuracy in dose rate determination, using the

148 infinite matrix assumption, has been questioned in general - and for this sample in particular - by

149 Guérin et al. (2012), especially when it comes to grain-size attenuation factors for uranium and thorium.

150 However, the exact value of the attenuation factors (constants) is not critical for our study since we are 
151

152

153

154

155

156

157

158

159

160

161

162

163

only interested in comparing the equivalent dose and dose rate distributions in this sample; we therefore used attenuation factors for beta dose rates from uranium and thorium. The effect of moisture on gamma dose rate was taken into account following Guérin and Mercier (2012), using the mean grain size of the sample and using the cubic-centred packing model. For the effect of moisture on beta dose rates, we used the water correction factors from Nathan and Mauz (2008) in sediments containing no carbonates, which were indirectly confirmed by Guérin and Mercier (2012). Here it should be noted however, that these correction factors have not been adapted to sand samples (for which the geometry of energy emission and absorption has consequences on the effect of moisture on beta dose rate - see Guérin et al., 2012). For the potassium feldspar extracts, the internal dose rate was calculated using dose rate conversion factors for potassium (Guérin et al., 2011) and the self-dose values from Guérin et al. (2012), and assuming an internal potassium content equal to $12.5 \pm 0.5 \%$ (Huntley and Baril, 1997). Finally, the contribution from Rb was calculated according to Readhead (2002) and Huntley and Hancock (2001).

\section{Grain size analysis and element composition}

Grain size analysis and single grain element composition were obtained from Scanning Electron Microscope (SEM) image analysis and Energy Dispersive Spectrometry (EDS), respectively. Guérin et al. (2012) already modelled dose rates in this sample but their study focused on average dose rates to the different grain-size classes. Nevertheless, the sample characteristics were taken from this previous study: the grain size distribution can be found in their Fig. 1 (where the frequency corresponds to the actual number of grains rather than the most commonly used mass fraction). The sample is a well-sorted medium sand, with a mean grain size of $360 \mu \mathrm{m}$ (geometric mean following Folk and Ward, 1957, calculated using the GRADISTAT program, Blott and Pye, 2001; in the following, all mean grain sizes are calculated accordingly). Based on EDS analysis, it is mainly (>99\% by number of grains) made up of three 
174 minerals: quartz (85\% of the grains), potassium (7\%) and sodium (8\%) feldspar. Single grain EDS analysis

175 further revealed that the grain-size distribution of potassium feldspar grains is similar to that of the 176 sample taken as a whole. The potassium concentration, calculated from the abundance of potassium

177 feldspar grains, and assuming a $12.5 \% \mathrm{~K}$ content of these feldspars (corresponding to the peak in the

178 histogram of $\mathrm{K}$ concentration from single grains, Fig. 2 in Guérin et al., 2012) is 1 \% by mass and

179 compares very favourably with gamma spectrometry results (Table 1).

\section{Sample preparation}

Prior to mineral separation, the sample was wet sieved to isolate $180-250 \mu \mathrm{m}$ sand grains. These

182 grains were then treated with $\mathrm{HCl}(10 \%)$ to remove carbonates, and with hydrogen peroxide $\left(\mathrm{H}_{2} \mathrm{O}_{2}\right)$ to 183 remove organic contaminants; despite a weak reaction, both treatments were continued until no

184 further reaction was visible. Two aqueous solutions of sodium heteropolytungstates (densities 2.58 and $\left.1852.62 \mathrm{~g} . \mathrm{cm}^{-3}\right)$ were used to isolate K-rich feldspar fractions $\left(<2.58 \mathrm{~g} . \mathrm{cm}^{-3}\right)$ and quartz $\left(>2.62 \mathrm{~g} . \mathrm{cm}^{-3}\right)$. The 186 quartz fraction was then etched with $\mathrm{HF}(40 \%)$ for 40 minutes to remove the outer portion of the grains 187 affected by alpha irradiation. After etching, any fluoride contaminants were removed by rinsing with $18810 \% \mathrm{HCl}$. This fraction was then re-sieved to $>180 \mu \mathrm{m}$ for further analysis, in particular for single grain measurements; this latter step removes any $<180 \mu \mathrm{m}$ grains resulting from the dissolution of residual feldspar in the quartz-rich fraction, or of small quartz grains.

\subsection{Luminescence instrumentation}

Grains were mounted in $9 \mathrm{~mm}$ base-diameter stainless steel cups using silicon oil. Aliquots of 6

$193 \mathrm{~mm}$ in diameter were measured for quartz, at the IRAMAT-CRP2A in Bordeaux, and of $\sim 3 \mathrm{~mm}$ in 194 diameter for feldspar extracts, at Ris $\varnothing$. Luminescence measurements were made using Ris $\varnothing$ TL/OSL DA19515 and DA-20 readers (Bøtter-Jensen et al., 2003; 2010); for quartz multi-grain aliquots, blue (470 nm) 196 light-emitting diodes (LED) were used with $7.5 \mathrm{~mm}$ Hoya U-340 detection filters; for feldspar, IR diodes 
emitting at $875 \mathrm{~nm}$ were used in combination with coupled Schott BG39 and Corning 7-59 detection

198 filters (transmission $320-460 \mathrm{~nm}$ ). Each ${ }^{90} \mathrm{Sr} /{ }^{90} \mathrm{Y}$ source was calibrated during the measurement period 199 by measuring several aliquots of calibration quartz irradiated with gamma rays (4.81 Gy; hereafter 200 referred to as Risø calibration quartz) from a national secondary-standard ${ }^{137}$ Cs source; this calibration 201 has been independently confirmed by Bos et al. (2006).

Single grains of quartz were measured using an automated Risø TL/OSL reader (DA 20) fitted 203 with a single grain attachment (Duller et al., 1999; Bøtter-Jensen et al., 2000). The grains were loaded into aluminium single-grain discs; each disc contains 100 holes $300 \mu \mathrm{m}$ in diameter and $300 \mu \mathrm{m}$ deep, on a $10 \times 10$ rectangular grid with $600 \mu \mathrm{m}$ spacing between centres. A green laser $(532 \mathrm{~nm})$ was used to stimulate these grains individually, with light detection through a $7.5 \mathrm{~mm}$ Hoya U-340 glass filter. To confirm that only one grain was loaded into each hole, the single grain discs were visually inspected using a microscope before measurement. Radiochromic films allowed the determination of a coefficient of variation of $5.6 \%$ in dose rates to individual positions on the single-grain disc (Lapp et al., 2012). Correcting for this spatial variation in dose rates to single grains did not significantly change the measured $D_{e}$ distributions, so we used a single beta source dose rate for all grain positions.

\subsection{Modelling: LSD algorithm and GEANT4}

Allison et al., 2006) is used to simulate the beta emission spectra from potassium feldspar grains (Fig. 1; such grains represent $7 \%$ of the total), and to track each primary (electron) and secondary (photon and

217 electrons) particle transport individually in a random close packing of spherical grains. The random close 218 packing is based on the Lubachevski-Stillinger-Donev (LSD) algorithm (Donev et al., 2005). The grain size 
thinly spread on a glass plate to ensure no grain overlap). The equivalent radius of the grains was

221 determined assuming spherical grains (by equivalent radius of a grain we mean the radius of a circle

222 whose surface would correspond to apparent, generally irregular surface of the grain). The compactness

223 of the sediment obtained by random packing of the grains, using the LSD algorithm, is 0.635; as a result,

224 the density of the medium when air fills the pore space, is calculated to be $1.68{\mathrm{~g} . \mathrm{cm}^{-3}}^{-3}$

The sample water content, as determined experimentally, is $12 \%$ - which corresponds to a

226 sediment density of $1.88 \mathrm{~g} \cdot \mathrm{cm}^{-3}$. To obtain the same density for the wet sediment in our Monte Carlo

227 simulations, air is replaced by uniform, 'light water' (with a density of $0.55 \mathrm{~g} . \mathrm{cm}^{-3}$ ) in pore spaces; this

228 leads to a calculated wet density for the simulated sediment equal to the experimental value. Here it

229 should be noted that these dry and wet sediment density values corresponding to the simulations are

230 close to 'typical' sediment densities such as those given e.g. by Aitken (1985, Appendix H). The low

231 density, uniformly distributed 'water' is an approximation; in practice, surface tension effects alter the

232 spatial distribution of water (density: $1 \mathrm{~g} . \mathrm{cm}^{-3}$ ) in the pore spaces - water forms thin layers at the

233 surface of grains and tends to accumulate where grains touch each other. Such modelling goes beyond

234 the scope of this study, however, it is difficult to say if a more realistic distribution of water would

235 significantly affect the results of the simulations. For charged particles, the stopping power (unit: $\mathrm{cm}^{2} \cdot \mathrm{g}^{-}$

$236{ }^{1}$ ) determines the energy loss in the media, so for example, energy loss in $10 \mu \mathrm{m}$ of water with a density

237 of $0.55 \mathrm{~g} . \mathrm{cm}^{-3}$ is equivalent to crossing $5.5 \mu \mathrm{m}$ of identical water but with a density of $1 \mathrm{~g} . \mathrm{cm}^{-3}$; one can

238 ignore here $4.5 \mu \mathrm{m}$ of air because of the negligible mass. As a result, in terms of energy loss in pore

239 space, the two scenarios are equivalent (light, uniformly distributed water, or dense, localised water and

240 air). However, some difference between the two cases will occur in terms of directional straggling; but

241 these are expected to even out on average. 
243 within the feldspar potassium grains. For simplicity, Guérin et al. (2012) simulated either pure potassium

244 feldspar grains (with a K content of 14\%, following stoichiometric values), or grains with zero potassium

245 content. This assumption allows simplification of the simulations; however, the continuous distribution

246 of $\mathrm{K}$ in the grains (cf. SEM-EDS analysis presented in Fig. 2 of Guérin et al., 2012) suggests that the actual

247 potassium distribution may be somewhat less heterogeneous than in the model. Here, it should be

248 noted that: (i) the potassium content of grains having a $\mathrm{K}$ content less than $6 \%$ are considered as zero

249 potassium grains; this is considered acceptable since these grains represent only $\sim 10-15 \%$ of the total

250 potassium in the sample; (ii) SEM-EDS analyses characterise only the surface of the grains, while the

251 beta dose rate originates in the entire volume (so SEM-EDS values might not be representative of the

252 content of the grains). We also observed low but non-zero values of $\mathrm{K}$ content from measurement of

253 quartz grains, implying that at least some $\mathrm{K}$ is residing on the surface of all grains. Thus, the number of

254 feldspar grains with intermediate $\mathrm{K}$ values is likely to be even lower than that observed in the data,

255 suggesting that our assumed binary distribution of K should have little influence on the validity of the

256 simulation results.

For tracking of both photons and electrons, Penelope physics datasets were used, as they are

258 well-adapted to the simulation of low energy electromagnetic interactions (Salvat et al., 2001).

259 Production cuts (i.e. range of secondary particles below which these secondary particles are not

260 generated) and maximum step size were set to $20 \mu \mathrm{m}$ to ensure accurate tracking down to one tenth of

261 the diameter of the dosimeter grains of interest. In other words, the energy that would be carried away

262 by a particle with a range of less than $20 \mu \mathrm{m}$ was assumed to deposit locally, and the interaction

263 probabilities were recalculated, by extrapolation of the provided Penelope datasets, every $20 \mu \mathrm{m}$ along

264 the particles tracks. To mimic infinite matrix conditions, a reflection algorithm was used (Nathan, 2011;

265 Guérin et al., 2012). 
Whereas in Guérin et al. (2012), the dose was only recorded in the grain-size classes of interest,

267 in this study every quartz grain in the range from 180 to $250 \mu \mathrm{m}$ in diameter is treated as an

268 independent dosimeter; this allows us to obtain beta dose rate distributions from potassium feldspar to

269 quartz grains. For each set of simulations (i.e. for each grain size distribution and potassium content),

270 ten different random close packing configurations were used. For each configuration, the emission and

271 tracking of $20,000,000$ primary particles were simulated at the calculation centre of the French National

272 Institute of Nuclear and Particle Physics (IN2P3). The uncertainties on the different numbers given in the

273 following are obtained by taking the standard errors on individual values from the 10 different simulated 274 configurations.

\section{Results}

\subsection{Multi-grain aliquots OSL, IRSL and age control}

For the inter-comparison sample studied here, the quartz OSL signal is dominated by the fast component. The SAR protocol (Murray and Wintle, 2000; 2003) was used with a preheat temperature of

$279200^{\circ} \mathrm{C}$, held for ten seconds, and a cutheat temperature of $180^{\circ} \mathrm{C}$ before test dose measurements. The 280 net signal intensity used in further calculations was derived from the sum of the OSL in the first $0.8 \mathrm{~s}$ of 281 stimulation minus a background signal (calculated from the following 2.4 s of stimulation, i.e. early 282 background subtraction). Nine aliquots were first exposed to a SOL 2 solar simulator for 3 hours and 283 then given a dose of $5 \mathrm{~Gy}$ in the luminescence reader. The measured to given dose recovery ratio $284(0.97 \pm 0.05)$ showed that our SAR protocol was well-suited to measure equivalent doses for this sample. 28521 equivalent doses were measured using multi-grain aliquots of quartz; the average recycling ratio was $2860.99 \pm 0.07$, and the resulting equivalent dose and age (4.73 $\pm 0.23 \mathrm{ka})$ are shown in Table 2 . equivalent dose is $6.90 \pm 0.30 \mathrm{~Gy}$. A g-value of $2.8 \pm 0.2 \% /$ decade was obtained from fading 
measurements performed on the same aliquots. Using the fading correction from Huntley and Lamothe

290 (2001), the resulting age of $4.28 \pm 0.27 \mathrm{ka}$ is in good agreement with the quartz OSL age, which confirms

291 that the quartz OSL signal was well reset at the time of deposition (cf. Murray et al., 2012). A post-IR

292 IRSL at $290{ }^{\circ} \mathrm{C}$ (pIR-IR 290 ; Thiel et al., 2011) dose of $13.7 \pm 0.6$ Gy was obtained from six different aliquots,

293 giving an apparent age of $6.69 \pm 0.36 \mathrm{ka}$. This age overestimation of $\sim 2 \mathrm{ka}$ is not surprising given the

294 young age of the sample since it is well-known that residual, difficult-to-bleach doses affect post-IR IRSL

$295 D_{e}$ determination from young samples. It corresponds to a residual dose of $\sim 6 \mathrm{~Gy}$ for this signal, which

296 fits within the variability of observed residual doses for well-bleached samples (i.e., samples sufficiently

297 exposed to sunlight to reset the quartz OSL signal; see, e.g., Buylaert et al., 2011). This further indicates

298 that the quartz OSL from this sample is most likely unaffected by poor-bleaching.

\subsection{Single grain OSL $D_{e}$ and dose rate distributions.}

The single grain $D_{e}$ measurements were all made using the SAR protocol with a preheat at 260

${ }^{\circ} \mathrm{C}$ for ten seconds, and a cutheat at $220{ }^{\circ} \mathrm{C}$ prior to test dose response measurement (note that thermal

302 transfer is negligible for this sample, $c f$. Nielsen et al., 2006). The net signal used in $D_{\mathrm{e}}$ calculations was

303 derived from the sum of the OSL in the first $0.05 \mathrm{~s}$ of stimulation minus a background signal (time

304 average of the last $0.2 \mathrm{~s}$; total stimulation time: $1 \mathrm{~s})$. Dose estimates from individual grains were

305 accepted if they passed the following rejection criteria (derived from Thomsen et al., 2005; 2007; 2012):

306 an error on the first test dose signal of less than $20 \%$ and a recycling ratio consistent with unity at two

307 standard deviations. Recuperation was negligible for all samples. Note that the purity of the quartz

308 extracts was examined on multi-grain aliquots using an IR-test (IRSL/BLSL ratios < 1\%; Murray et al.,

309 submitted).

Fig. 2 shows the relationship between the first ('natural') test dose signal and measured 
312 (batch 54 , heated and then given a $4.81 \mathrm{~Gy}$ dose using a secondary-national standard ${ }^{137} \mathrm{Cs}$ source in

313 scatter free-geometry, Fig. 2a), (ii) from fractions of quartz from the inter-comparison sample exposed

314 to a solar simulator for three hours and then given gamma doses of respectively $1.92,4.81$ and $9.62 \mathrm{~Gy}$

315 (Figs. 2b, c ,d), and (iii) from natural quartz from the inter-comparison sample (Fig. 2e).

\subsubsection{Single grain gamma dose distributions}

Table 3 lists a number of statistical characteristics of the equivalent dose distributions of figure

2, and resulting $D_{e}$ measurements derived using different statistical models: the Central Age Model

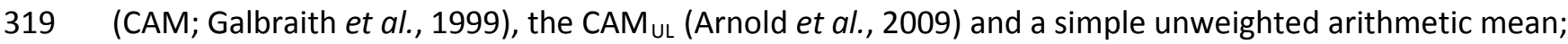

320 where relevant, dose recovery ratios are also given; all dose recovery ratios are within $10 \%$ of unity.

321 Furthermore, they are all consistent with unity, within two standard errors (except for the 1.92 Gy dose

322 recovery test, where the $\mathrm{CAM}_{\mathrm{UL}}$ gives a measured to given dose ratio equal to $0.93 \pm 0.03$ ).

The Risø calibration quartz and the inter-comparison sample show different average

luminescence intensities in response to a fixed test dose of $2.2 \mathrm{~Gy}$ (first test dose signal). Furthermore,

the average luminescence intensity of the signals induced by gamma irradiations in dose recovery

experiments depends on the given dose. As a consequence, the average relative uncertainties on

327 individual dose estimates vary between the different samples: $13 \%$ for the Ris $\varnothing$ calibration quartz (given

328 dose: $4.81 \mathrm{~Gy}$ ) and $27 \%, 21 \%$ and $13 \%$ for the inter-comparison sample for given doses of $1.92,4.81$

329 and $9.62 \mathrm{~Gy}$, respectively (see Table 3). However, the relative overdispersion (OD) values from the CAM

330 show little variation between Ris $\varnothing$ calibration quartz and the inter-comparison sample, or as a function

331 of dose for the latter (16\% on average; cf. Table 3 ); the different OD values for the gamma dose

332 recovery experiments are statistically indistinguishable, which confirms the pattern seen by Thomsen et

333 al. $(2007 ; 2012)$ in the low dose region. Similar conclusions can be drawn for the CAM $\mathrm{UL}_{\mathrm{L}}$, when the

334 absolute OD (in Gy) is expressed as a fraction of the central dose. Fig. $\mathbf{3}$ shows a standardised residual 
analysis in the form of quantile-quantile plots (see Galbraith and Roberts, 2012, for other examples of

336 such plots and their discussion). Quantile-quantile plots can be used to visually assess the normality of

337 the distribution of residuals from the models. The standardised residuals $\left(\left(d_{i}-\delta\right) / \sigma_{i}\right.$, where $d_{i}$ is the $i^{\text {th }}$

338 measurement of dose, $\sigma_{i}$ its associated uncertainty - i.e., the quadratic sum of the analytical uncertainty

339 and the overdispersion - and $\delta$ is the central value determined with the model) are sorted and plotted

340 against the estimates expected from a normed and centred Gaussian distribution. The 1:1 line indicates

341 the expected fit to the data if residuals are normally distributed.

Interestingly, from Fig. $\mathbf{3}$ it can be seen that for the gamma dose distributions, the standardised

residuals from both the CAM and the $\mathrm{CAM}_{U \mathrm{~L}}$ are consistent with a normal distribution, i.e. the observed

344 residuals plotted against a normal distribution fall on a 1:1 line, despite a few outliers in the tail regions.

345 In other words, it appears that the intrinsic overdispersion can be well described either by the same 346 relative or the same absolute uncertainty; this makes the choice between normal and lognormal age 347 models arbitrary at this stage.

One of the differences between laboratory gamma dosed and the natural $D_{e}$ distributions lies in the different dose rates to which individual quartz grains have been exposed in sedimentary media. Fig. 4 shows the results of the GEANT4 simulations of the single-grain beta dose rate distribution from

352 potassium feldspar grains for the inter-comparison sample. This distribution is positively skewed

353 (skewness: 1.07) and can be fitted by a lognormal distribution (red line), which is in agreement with 354 previously published work (Mayya et al., 2006). The positive skewness can be understood as a result of 355 few quartz grains being close to potassium feldspar grains (high dose rate tail of the distribution), 356 whereas most quartz grains are at some distance - compared to the range of beta particles - from beta 357 radioactive sources (mode of the distribution). The distribution has a relative standard deviation of 
$31.2 \pm 1.4 \%$. Note that the presence of hot-spots does not lead to any distinguishable bi-modality in the

359 resulting dose rate distribution.

Grain to grain variations in gamma and cosmic dose rates can reasonably be assumed to be negligible in this sample, given the range of these radiations (>tens of $\mathrm{cm}$ ). We assume that there is no

362 other source of dispersion in beta dose rates to quartz grains - which is difficult to prove but supported 363 by the absence of heavy minerals such as zircons, apatites, etc. from the SEM-EDS analysis (these could 364 be potential sources of uranium and thorium). As a consequence, the relative standard deviation of the total dose rates to single grains of quartz $(15.6 \pm 0.7 \%)$ is obtained by multiplying the dispersion in beta dose rates from potassium by the relative contribution of this component to the total (50\%).

\subsubsection{Over-dispersion in the inter-comparison natural sample}

Equivalent doses measured for the natural portion of the inter-comparison sample are plotted against natural test dose responses in Fig. 2e. Standardised residual analyses from the CAM and the $\mathrm{CAM}_{U L}$ are shown in Fig. 5. As for the gamma dosed populations, both models provide good fits to the experimental data, and the resulting equivalent doses are consistent with each other. quantified at this stage: (i) an intrinsic OD (i.e. the OD resulting from the measurement protocol; in

374 other words we regard this intrinsic OD to originate from unrecognised/unquantified uncertainties 375 inherent in the measurement, rather than as so-called 'natural variations in the OSL properties' giving 376 rise to different true equivalent doses - cf. Galbraith et al., 2005). Our best estimate of this is

377 determined from the gamma dose recovery tests in the dose range of interest (section 4.2.1); and (ii) an 378 extrinsic OD (i.e. the OD resulting from all environmental factors external to the grains, such as the 379 degree of light exposure before burial and grain-to-grain variations in dose rate). In this sample, we 
consider the extrinsic OD to be dominated by the dispersion in dose rates (section 4.2.2), since we are

381 confident that the sample was well bleached at deposition.

In this sample, we have determined the intrinsic OD from the CAM ( $15 \pm 3 \%$ at $\sim 5$ Gy) and the

383 standard deviation in dose rates $(15.6 \pm 0.7 \%)$; these can be summed quadratically to give a minimum

384 estimate of OD that should be observed in the natural sample, of $22 \pm 3 \%$. This compares very favourably

385 with the measured OD ( $23 \pm 2 \%)$; thus, it seems that the natural OD for the well-bleached inter-

386 comparison sample can be fully explained by two contributions: the intrinsic OD and the dispersion in

387 dose rates.

\subsection{Factors influencing the dispersion in dose rates}

Given that it appears that dose rate variations contribute about $50 \%$ to the total OD in our

sample, it is now useful to investigate the factors influencing the dispersion in dose rate to single grains.

391 This was done by varying several parameters of the GEANT4 model. Mayya et al. (2006) have already

392 shown the effect of average potassium concentration on dose rate distributions in sands where

393 potassium is located in potassium-rich feldspar grains: the skewness and dispersion of dose rate

394 distributions increase as the number of potassium-rich grains is decreased (relative to the number of

395 quartz grains). This can be understood by considering that the average distance between source and

396 dosimeter grains is increased as the potassium content is decreased because of a reduction in the

397 number of feldspar grains; as a result, fewer quartz grains are close to potassium sources and most are

398 at a distance from any source. Similarly, one would then also expect that the average grain size of the

399 sediments would have a similar effect on dose rate distributions: as the grain size is increased, the

400 distances between source and dosimeter grains is also increased, which should lead to more skewed

401 and more dispersed distributions. 
Two parameters were thus varied in the simulations: firstly, the potassium content was varied

403 by changing the fraction of potassium-rich feldspar grains, while keeping the grain size distributions

404 similar for quartz and feldspar; secondly, the grain size distributions of the whole sample were

405 multiplied by different scaling factors, so that the sorting of the sediments remained untouched but the

406 simulated grain size distributions went from fine/very fine sands up to medium/coarse sands (Fig. 6).

407 Fig. 7 shows frequency histograms of beta dose rate distributions to quartz grains for various grain size

408 distributions and average matrix (bulk) potassium concentrations. The relative standard deviation of

409 these distributions is plotted in Fig. 8, for different grain sizes, as a function of average potassium

410 content. As expected, the relative dispersion increases when the potassium content is decreased and/or

411 when the grain size is increased, up to $135 \pm 19 \%$ for a mean grain size of $637 \mu \mathrm{m}$ with a $\mathrm{K}$ content of

$4120.14 \%$

We have compared our results, in terms of relative standard deviation in beta dose rates from

414 potassium feldspar, with those presented by Mayya et al. (2006) in their Fig. 4. For 1\% potassium and a

415 unique grain size $(200 \mu \mathrm{m})$, Mayya et al. (2006) found a relative standard deviation of 28\%; for a mean

416 grain size of $255 \mu \mathrm{m}$, we found $20 \%$ and only $9 \%$ for $149 \mu \mathrm{m}$. It is not straightforward to understand

417 these differences, partly because the two approaches are so different (in particular, Mayya et al.

418 focused on determining the minimum dose due to the presence of hotspots: Morthekai, Pers. Com.),

419 and parameters may have different values. For example, the emission of beta particles in their paper is

420 considered to be point-like, whereas in the Monte Carlo simulations the initial position within the

421 emitting grains is sampled homogeneously. Straggling effects are taken into account in our Monte Carlo

422 modelling, but not in Mayya et al. (2006). Furthermore, in their paper the minimum distance between a

423 quartz grain and the closest hotspot - defined as the distance between the centres of the two

424 corresponding grains (Morthekai, Pers. Com.) - is 0; in other words, two grains can overlap, which is

425 physically unrealistic. This may seem to be negligible, but it most likely explains the important high dose 
tails in their Fig. 3. $b$ (it should be emphasized that this did not affect the minimum dose due to the

427 presence of hotspots). Finally, there is an apparent peak in the dose distributions for very low doses (cf.

428 Fig. 3a), which according to the original authors is a numerical artefact (Morthekai, Pers. Com.); this

429 could contribute to the relative standard deviation in dose rates. It is very difficult to know at this stage

430 if one or more of these factors can explain the difference between our results and those from Mayya et

431 al. (2006). Nonetheless, despite these differences, the tendencies observed when parameters are varied

432 (in particular potassium content) remain the same. Because Monte Carlo simulations have fewer

433 approximations and closely mimic nature and because we model a more representative sediment

434 matrix, we tend to believe that the results of our simulations are better representative of the deviation

435 in dose rates due to the presence of hotspots compared to the approximate analytical treatment in

436 Mayya et al. (2006).

It should be emphasised here that, for a given potassium content and assuming that beta dose

438 rates from potassium are the only source of grain to grain dose rate variations, the dispersion on total

439 dose rates to quartz grains will decrease as the total dose rate is increased; this is because the relative

440 contribution to dose rate from potassium is decreased. In other words, the dispersion values from Fig. 8

441 should always be scaled by the relative contribution of beta dose rates from potassium to the total. In a

442 comprehensive study of more than 4,000 sediment samples from various contexts and geographical

443 locations, Ankjærgaard and Murray (2007) have shown that beta dose rates account on average for

$444 \sim 67 \%$ of the total dose rates (when working on sand-sized grains previously etched with concentrated

$445 \mathrm{HF}$, i.e. not accounting for any alpha dose rate contribution). Moreover, for $95 \%$ of the samples, ${ }^{40} \mathrm{~K}$

446 contributed between 40 and $92 \%$ of the total beta dose rate. In other words, the contribution to the

447 total dose rate to quartz grains from the beta dose rate derived only from potassium ranged from 27 to

$44862 \%$ in almost all cases. Fig. 9 shows the modelled dispersion in total quartz dose rates as a function of 449 potassium content for three samples in each of which the total dose rate is fixed (at 1, 2 and 3 Gy.ka ${ }^{-1}$ ). 
450

451

452

453

454

455

456

457

458

459

460

461

462

463

464

465

466

467

468

469

470

471

The known likely range of potassium-derived beta dose rate contribution to the total ( 27 to $62 \%$, derived above) are shown as dashed lines in Fig.9; these indicate the likely standard deviations to be expected for well-sorted sands of different mean grain sizes, for typical K concentration.

\section{Discussion}

\subsection{Typical OD for well-bleached samples}

Many age models (FMM, MAM, IEU) require the input of an OD value before the model can be used to identify a representative dose component(s). Determining an accurate OD representative of a well-bleached population is, therefore, at the heart of most single-grain studies. Sometimes, the intrinsic OD determined by a dose recovery experiment is used as a minimum value (e.g., Thomsen et al., 2007); in other studies the OD is allowed to vary between fixed values (e.g., Jacobs et al., 2008b).

The results from our Monte Carlo simulations show that a typical OD value for a well-bleached sample will depend on grain size, potassium content, and total dose rate. Considering the effect of potassium feldspar grains on dispersion in single-grain dose rates, there is no a priori limit on the OD of a natural sample and certainly no typical OD for well-bleached samples. This could explain the wide range of ODs observed in natural samples presumed to be well-bleached (but presumably affected by beta dose rate heterogeneities, see Fig. 1 of Thomsen et al., 2012, and references therein; see also, e.g., Jacobs, 2010; Jacobs et al., 2011; 2012; 2013; Gliganic et al., 2012).

\subsection{When to use the dose rate model}

It should be emphasised that the model presented here is expected to be used in cases where single grain dose rates need to be simulated to disentangle different sources of OD in single grain $D_{e}$ measurements. In this study, the model successfully explains the discrepancy between the observed OD in the natural $D_{e}$ distribution and the intrinsic $O D$ resulting from the measurement protocol, for a single 
472 sample. It is difficult to predict how well it might perform on a variety of samples. Nevertheless, our

473 understanding of the processes involved allows us to be confident that dispersion in beta dose rates

474 arising from the distribution of potassium will be most important when the average grain size is in the

475 sand and gravel range (rather than silt or clay), the potassium content is low $(<1 \%)$, and the total dose

476 rate is small $\left(<1 \mathrm{~Gy} \cdot \mathrm{ka}^{-1}\right)$.

$477 \quad$ 5.3. Implications for the use of different age models

Interestingly, in the literature so-called age models (e.g., CAM, MAM, FMM) are actually dose

479 determination models. Very few studies focus on dose rates during burial, even fewer consider dose

480 rate distributions in the analysis of single-grain dose distributions. The simulation results presented in

481 this study raise important questions concerning how luminescence ages are calculated. In particular, it is

482 not clear how individual data should be weighted; each single grain equivalent dose estimates is not

483 measured with the same precision, and each grain has received a different unknown dose rate; thus a

484 dose distribution is not equivalent to an age distribution.

In the ideal case of a single 'true dose' (i.e., every grain has absorbed the same dose), the central

486 dose (in this section, by central dose we mean the value most appropriate for use with an average dose

487 rate to derive an age) is commonly derived using the logged or the unlogged central age model (see

488 section 2). An alternative approach, commonly used in multi-grain analyses, is to use the unweighted

489 arithmetic mean; this approach discards analytical uncertainties on individual dose estimates on the

490 grounds that these uncertainties are trivially small compared to the variability in $D_{e}$ measurements. The

491 use of this latter approach inherently implies that the main source of dispersion is unknown and is much

492 bigger than all known sources of analytical uncertainty.

The intrinsic dispersion in the $D_{e}$ data can generally be equally well described by normal and

494 lognormal distributions. However, the dose rate distribution from potassium feldspar grains is 
positively skewed and can only be best described by a lognormal distribution; each individual dose

496 component of this distribution is sampled in the lab as a normal or a log normal distribution. In many

497 cases dose recovery distributions can be adequately fitted using the CAM (which assumes a log normal

498 distribution); this has been demonstrated here by the Gaussian distribution of the residuals from the

499 CAM (Fig.3 - right panel), using the inter-comparison sample. Given that log normal distributions can

500 describe both the natural dispersion arising from dose rates, and the measurement induced dispersion

501 in the data, suggests that the use of logged models best represent $D_{e}$ distributions in natural samples,

502 and so provide the best estimates of OD.

The question is how best to determine the burial dose from a distribution of single grain $D_{e}$

504 values, in the absence of the knowledge of the underlying dose rate distribution. Almost all dose rates

based on high resolution gamma spectrometry, Neutron Activation Analysis (NAA), beta counting or any

506

other analytical technique are arithmetic means of repeated measurements of the spatially averaged

507 radioactivity in the sample. Thus, we typically know only an average dose rate in the sample. The dose

508 distribution on the other hand is known at the single grain level and different measures of central

509 tendency can be applied to derive a representative dose. If an age is derived by dividing a geometric

510 mean $D_{e}$ by an arithmetic mean dose rate, then the age is likely to be underestimated to some degree

511 (since unweighted geometric means are systematically lower than unweighted arithmetic means). For

512 example, consider a sample in which the distribution of dose rates dominates the natural dispersion.

513 Suppose this to be a well-bleached, well-behaved sample in which the uncertainty on the measurement

514 of dose is negligibly small (e.g., 1\%). All grains must by definition record the same age. First, consider a

$51510 \mathrm{ka}$ old fine-grain sample with a uniform dose rate of $1 \mathrm{~Gy} / \mathrm{ka}$. We measure 3 grains, each with a dose

516 of $10 \mathrm{~Gy}$ : the average age is $10 \mathrm{ka}$ and the CAM age is $10 \mathrm{ka}$ with no overdispersion. Now let us consider

517 a less homogeneous (coarse grained) sample, of the same age and average dose rate, from which we

518 sample three representative grains which have experienced dose rates of $0.6,0.9$ and $1.5 \mathrm{~Gy} / \mathrm{ka}$ 
520 distribution is positively skewed, and the individual grain ages of course remain at $10 \mathrm{ka}$; the average

521 age is 10 ka but the CAM age (using a geometric mean of $D_{e}$ values) is 9.3 ka. Thus, it appears that if the

522 scatter in the measured equivalent dose distribution arises primarily as the result of grain to grain

523 variability in the dose rates (which is much larger than the intrinsic variability due to measurement), and 524 one measures an average dose rate, then it is more appropriate to use the simple mean rather than the 525 geometric mean $\mathrm{D}_{\mathrm{e}}$.

More generally, the equivalent dose derived from measured $D_{e}$ distributions using any age

527 model should be as close as possible to the average of the true underlying dose rate distribution.

528 Sometimes, this will be best estimated using the CAM (or geometric mean); for instance, when all grains

529 have received the same dose and the dominant source of dose dispersion is multiplicative error

530 properties. However, in those cases where the dose rate distribution is unknown but the natural dose

531 distribution is considerably overdispersed compared to a (gamma) dose recovery experiment, it seems

532 reasonable to assume that the dominant source of dispersion is dose rate (in any case, it is unlikely to be

533 explained by multiplicative error properties, because these should be entirely accounted for by the dose

534 recovery experiments). In such cases the central dose may be best estimated by using an unweighted

535 arithmetic average; the CAM will bias the results to give an equivalent dose inappropriate to the average

536 dose rate, and so an underestimate of the age. Obviously, this approach has the drawback that one

537 gives equal weight to individual $D_{e}$ values that are known with different degrees of precision.

To some extent, the above problem could be circumvented if a geometric mean dose rate was

539 available. However, only direct measurement of single-grain dose rate distributions would allow the 540 calculation of geometric mean dose rates; in general, such data is not obtainable experimentally. For the 541 inter-comparison sample, the geometric mean of the GEANT4 simulated single-grain beta dose rates 
542 from potassium is $\sim 5 \%$ lower than the arithmetic mean; in the absence of uncertainties on $D_{e}$

543 measurements (or if they are negligible compared to other sources of dispersion), using a geometric

544 mean to calculate a central dose (e.g., CAM) together with an average dose rate would thus result in an

545 age underestimation of $\sim 2 \%$ compared to that using the arithmetic mean for equivalent dose and dose

546 rate (since beta dose rate from potassium contributes $\sim 50 \%$ of the total). In a worst case scenario, the

547 difference between geometric and arithmetic means of beta dose rates from potassium could be as

548 much as $50 \%$ (based on a simulation assuming $[\mathrm{K}]=0.14 \%$, mean grain size of $637 \mu \mathrm{m}-\mathrm{cf}$. Fig. 8). Then

549 the discrepancy arising from the division of a geometric mean equivalent dose estimate by an average

550 dose rate would result in an age underestimation of $25 \%$. Although this effect depends on potassium

551 content, grain size and total dose rate, it should be noted that the consequence of using a geometric

552 mean is a systematic age underestimation, not a random variation about some mean. In absence of

553 modelled data such as presented in this study (or a better analytical approach), it appears that

554 arithmetic means of $D_{e}$ should give us the most accurate age.

\subsection{A case study: the inter-comparison sample}

In this sub-section, we discuss in practice the choice of an appropriate analysis model for $D_{e}$

557 calculation, following the simulation results obtained for the inter-comparison sample.

In the natural $D_{e}$ distribution of the well-bleached inter-comparison sample, three main factors

559 contribute to variations in $D_{e}$ estimates, each with similar magnitudes: known measurement

560 uncertainties, an intrinsic source of scatter in single-grain doses (measureable, but of unknown origin;

561 Thomsen et al., 2005; Galbraith et al., 2005), and finally dose rate variations. Fig. 10 shows the

562 relationship between the absolute (Fig. 10a) or relative (Fig. 10b) errors and individual dose estimates

563 for the gamma dose recovery distribution obtained using the inter-comparison sample. The data are

564 highly scattered, and no clear trend is observable; if anything, there may be a weak tendency for the 
relative uncertainties to decrease with dose. Both the CAM and $\mathrm{CAM}_{\mathrm{UL}}$ indicate that the data are

566 overdispersed, with a magnitude similar to the dispersion of measurement uncertainties. Finally, Fig. 10

567 (c, d) shows the same plots of uncertainty as a function of dose for the distribution of $D_{e}$ estimates from

568 the natural inter-comparison sample. Again there is no unique trend, although overdispersion is

569 increased presumably due to variations in external beta dose rates. In all four cases it is difficult to

570 ascertain which age model, i.e. CAM, $\mathrm{CAM}_{\cup L}$ or unweighted arithmetic average, to use.

It is interesting to note that experimentally, for the equivalent dose populations investigated in

572 this study, the CAM-based equivalent doses are greater than CAM $\mathrm{CL}_{\mathrm{L}}$ results (Table 3), because - even

573 though there is no clear trend in the graphs from Fig. 10 - average relative uncertainties appear to

574 decrease slightly with increasing dose (thus placing more weight on higher dose estimates when using

575 CAM), and inversely for absolute doses (with the consequence of lowering CAM $\mathrm{CL}_{\mathrm{L}}$ central dose).

The fact that we measure non-zero intrinsic OD values tells us that we underestimate our

577 uncertainties on individual $D_{e}$ estimates, possibly due to variability in grain to grain natural OSL

578 properties that is not accounted for by analytical uncertainties. When applying our GEANT4 based dose

579 rate model, the only source of systematic variation in $D_{e}$ distributions that we know of - albeit only

580 through modelling - is the variation in dose rates to individual grains; it is difficult to justify using this

581 source of dispersion in the weighting of De estimates (as is the case when using e.g. the CAM), as dose

582 rate variability is independent of uncertainties of dose estimates. We may then decide to simply ignore

583 our uncertainties in central $D_{e}$ estimations and calculate unweighted averages of equivalent doses. Not

584 only are the dose recovery tests satisfactory (cf. Table 3), but doing so we would also compare

585 arithmetic means of equivalent doses with arithmetic means of dose rate. In the case of the inter-

586 comparison sample, (i) the three identified sources of dispersion in $D_{e}$ (analytical uncertainties, intrinsic

587 and extrinsic OD) are of comparable sizes and (ii) the intrinsic OD is reasonably well-fitted by both 
normal and lognormal distributions of corresponding uncertainties (cf. standardised residual analyses of

589 the CAM and the $\mathrm{CAM}_{\mathrm{UL}}$ ); it is not surprising that all three models (including an unweighted arithmetic average) give a set of consistent ages. In general, we argue that only a careful analysis of the sources of dispersion can lead to an informed decision regarding the most appropriate $D_{e}$ determination model on 592 a sample by sample basis.

Our study has not considered issues related to changes in OSL sensitivity of the grains between nature and laboratory irradiations; any such changes can be a source of additional intrinsic overdispersion in $D_{e}$ populations (undetected by gamma dose recovery experiments; see, e.g., Stokes, 1994a, b; Singhvi et al., 2011). But given that there is a satisfactory agreement between the observed

597 OD and the predicted OD (from intrinsic OD and beta dose rate variation), we think this sensitivity change effect must be negligible. From a dose rate perspective, the presence of highly radioactive minerals such as, e.g., zircons will also induce additional extrinsic over-dispersion. Our Geant4 model was designed to describe the effect of potassium feldspar grains on extrinsic over-dispersion, because 601 this was considered the most likely and most important source - at least for beta dose rates. Nevertheless, similar models using the same architecture can be used to predict the effect of uranium and thorium sources of any given geometry and size distributions.

\section{Conclusion}

We have developed a new model to quantify the effect of grain size and potassium concentration (feldspar hotspots) on the grain-to-grain dose rate variations for well sorted sediments. The model is successfully tested using experimental data obtained from a well characterised sediment sample, and predictions are made for other sediments with similar sorting but different grain sizes and $\mathrm{K}$ concentrations. The model provides estimates of minimum expected extrinsic overdispersion for various grain size distributions, potassium contents and total dose rates. These estimates, together with an 
611 analysis of over-dispersion in laboratory dose recovery data (intrinsic overdispersion), allow us to

612 investigate the sources of variability in equivalent dose measurements from individual grains in our test

613 sample. It is shown that consideration of beta dose rate variability has an important bearing on the use

614 of statistical models such as CAM, MAM, FMM, IEU, etc. for deriving the representative equivalent dose

615 that corresponds to the average dose rate estimate. Furthermore, our results imply that, for well

616 bleached samples, unweighted arithmetic mean dose together with the average dose rate may provide

617 a more accurate estimation of age, particularly in cases where the dispersion in measured $D_{e}$ values is

618 dominated by extrinsic over-dispersion rather than measurement uncertainties. This conclusion has

619 important implications for the analysis of more complicated dose distributions affected by incomplete

620 bleaching and post-depositional mixing.

621

622 Acknowledgements

623 This work was funded by a H.C. Ørsted postdoctoral grant awarded to GG. Monte Carlo simulations were

624 performed at the Calculation Centre of the French National Institute for Nuclear and Particle Physics

625 (CCIN2P3) via the TGE Adonis/TGIR Huma-Num. The authors are grateful to two anonymous reviewers

626 for contsructive and useful comments on an earlier version of this article.

627 References

628 Agostinelli S., et al. (Geant4 Collaboration) (2003). Geant4 - a simulation toolkit. Nuclear Instruments 629 and Methods A, 506, 250-303.

630 Allison J., et al. (Geant4 Collaboration) (2006). Geant4 developments and applications. IEEE Transactions 631 on Nuclear Sciences, 53, 270-278.

632 Ankjærgaard, C., Murray, A.S., 2007. Total beta and gamma dose rates in trapped charge dating based 633 on beta counting. Radiation Measurements 42, 352-359.

634 Arnold, L. J., Roberts, R. G., Galbraith, R. F., DeLong, S. B., 2009. A revised burial dose estimation 635 procedure for optical dating of young and modern-age sediments. Quaternary Geochronology 4, 306636325.

637 Blott, S.J., Pye, K., 2001. Gradistat: a grain size distribution and statistics package for the analysis of 638 unconsolidated sediments. Earth Surface Processes and Landforms, 26, 1237-1248. 
Bos, A.J.J., Wallinga, J., Johns, C., Abellon, R.D., Brouwer, J.C., Schaart, D.R., Murray, A.S., 2006. Accurate calibration of a laboratory beta particle dose rate for dating purposes. Radiation Measurements, 41, 1020-1025.

Brennan, B. J. 2006. Variation of the alpha dose rate to grains in heterogeneous sediments. Radiation Measurements 41, 1026-1031.

Brennan, B. J., Schwarcz H. P., Rink J., 1997. Simulation of the gamma radiation field in lumpy environments. Radiation Measurements 27, 2, 299-305.

Buylaert, J.-P., Ankjaergaard, C., Murray, A.S., Nielsen, A., 2006. A proposed laboratory intercomparison sample based on a beach-ridge sand from Skagen (Denmark), poster. In: UK-LED Meeting, Liverpool.

Buylaert, J-P, Thiel, C, Murray, A.S., Vandenberghe, D.A.G., Yi, S., Lu, H., 2011. IRSL and post-IR IRSL residual doses recorded in modern dust samples from the Chinese Loess Plateau. Geochronometria, 38, 432-440.

Bøtter-Jensen, L., Bulur, E., Duller, G.A.T., Murray, A.S., 2000. Advances in luminescence instrument systems. Radiation Measurements 32, 523-528.

Bøtter-Jensen, L., Andersen, C.E., Duller, G.A.T., Murray, A.S., 2003. Developments in radiation, stimulation and observation facilities in luminescence measurements. Radiation Measurements 37, 535541.

Bøtter-Jensen, L., Thomsen, K.J., Jain, M., 2010. Review of optically stimulated luminescence (OSL) instrumental developments for retrospective dosimetry. Radiation Measurements, 45, 253-257.

Chauhan, N., Singhvi, A., 2011. Distribution in SAR palaeodoses due to spatial heterogeniety of natural beta dose. Geochronometria, 38, 190-198.

Cunningham, A. C., DeVries, D. J., Schaart, D. R., 2012. Experimental and computational simulation of beta-dose heterogeneity in sediment. Radiation Measurements, 47, 1060-1067.

Donev A., Stillinger F. H., Torquato S., 2005. Neighbor List Collision-Driven Molecular Dynamics Simulation for Nonspherical Particles. I. Algorithmic Details II. Applications to Ellipses and Ellipsoids, Journal of Computational Physics, 202(2), 737-764 (part I) and 765-793 (part II).

Duller, G.A.T., Bøtter-Jensen, L., Murray, A.S., Truscott, A.J., 1999. Single grain laser luminescence (SGLL) measurements using a novel automated reader. Nuclear Instruments and Methods B, 155, 506-514.

Folk, R.L., Ward, W.C., 1957. Brazos River bar: a study in the significance of grain size parameters. Journal of Sedimentary Petrology, 27, 3-26.

Galbraith, R. F., Green, P. F., 1990. Estimating the component ages in a finite mixture. Nuclear Tracks and Radiation Measurements 17, 196-206.

Galbraith, R.F., Roberts, R.G., 2012. Statistical aspects of equivalent dose and error calculation and display in OSL dating: an overview and some recommendations. Quaternary Geochronology 11, 1-27.

Galbraith, R., Roberts, R.G., Laslette, G., Yoshidda, H., Olley, J., 1999. Optical dating of single and multiple grain quartz from Jinmium rock shelter, northern Australia. Part I. Experimetal design and statistical models R.F. Archaeometry 41, 339-364. 
676

677

678

679

680

681

682

683

684

685

686

687

688

689

690

691

692

693

694

695

696

697

698

699

700

701

702

703

704

705

706

707

708

709

710

711

712

713
Galbraith, R.F., Roberts, R.G., Yoshida, H., 2005. Error variation in OSL palaeodose estimates from single aliquots of quartz: a factorial experiment. Radiation Measurements 39, 289-307.

Guérin, 2011. Modélisation et simulation des effets dosimétriques dans les sédiments quaternaires : application aux méthodes de datation par luminescence. PhD thesis. Université Bordeaux 3.

Guérin, G., Mercier, N. and Adamiec, G, 2011. Dose rate conversion factors: update. Ancient TL, 29 (1), 5-8.

Guérin, G., Mercier, N., 2012. Preliminary insight into dose deposition processes in sedimentary media on a grain scale: Monte Carlo modelling of the effect of water on gamma dose rates. Radiation Measurements, 47, 541-547.

Guérin, G., Mercier, N., Nathan R., Adamiec, G., Lefrais, Y., 2012. On the use of the infinite matrix assumption and associated concepts: a critical review. Radiation Measurements, 47, 778-785.

Guérin, G., Murray A. S., Jain M., Thomsen K. J., Mercier, N., 2013. How confident are we in the chronology of the transition between Howieson's Poort and Still Bay? Journal of Human Evolution 64, 314-317.

Huntley D.J., Lamothe M., 2001. Ubiquity of anomalous fading in K-feldspar and the measurement and correction for it in optical dating. Canadian Journal of Earth Sciences 38, 1093-1106.

Jacobs Z., 2010. An OSL chronology for the sedimentary deposits from Pinnacle Point Cave 13B-A punctuated presence. Journal of Human Evolution 59, 289-305.

Jacobs, Z., Roberts, R.G., Galbraith, R.F., Deacon, H.J., Grün, R., Mackay, A., Mitchell, P., Vogelsang, R., Wadley, L., 2008a. Ages for the Middle Stone Age of southern Africa: implications for human behavior and dispersal. Science 322, 733-735.

Jacobs, Z., Wintle, A.G., Roberts, R.G., Duller, G.A.T., 2008b. Equivalent dose distributions from single grains of quartz at Sibudu, South Africa: context, causes and consequences for optical dating of archaeological deposits. Journal of Archaeological Science 35, 1808-1820.

Jacobs, Z., Meyer, M.C., Roberts, R.G., Aldeais, V., Dibble, H., El Hajraoui, M.A., 2011. Single-grain OSL dating at La Grotte des Contrebandiers ('Smugglers' Cave'), Morocco: improved age constraints for the Middle Paleolithic levels. Journal of Archaeological Science 38, 3631-3643.

Jacobs, Z., Roberts, R.G., Nespoulet, R., El Hajraoui, M.A., Debénath, A., 2012. Single grain OSL chronologies for Middle Palaeolithic deposits at El Mnasra and El Harhoura 2, Morocco: implications for Late Pleistocene human-environment interactions along the Atlantic coast of northwest Africa. Journal of Human Evolution 62, 377-394.

Jacobs, Z., Hayes E. H., Roberts, R.G., Galbraith, R. F., Henshilwood, C.S., 2013. An improved OSL chronology for the Still Bay layers at Blombos Cave, South Africa: further tests of single-grain dating procedures and a re-evaluation of the timing of the Still Bay industry across southern Africa. Journal of Archaeological Science 40, 579-594.

Jain, M., Thomsen, K.J., Bøtter-Jensen, L., Murray, A.S., 2004. Thermal transfer and apparent-dose distributions in poorly bleached mortar samples: results from single grains and small aliquots. Radiation Measurements 38, 101-109. 
Lapp, T., Jain, M., Thomsen, K. J., Murray, A. S. \& Buylaert, J. P., 2012. New luminescence measurement facilities in retrospective dosimetry. Radiation Measurements 47, 803-808.

716 Lepper, K., 2001. Development of an objective dose distribution analysis method for OSL dating and pilot 717 studies for planetary applications. Ph.D. Thesis, Oklahoma State University, Stillwater.

718 Mayya, Y.S., Morthekai, P., Murari, M.K., Singhvi, A.K., 2006. Towards quantifying beta microdosimetric

719 effects in single-grain quartz dose distribution. Radiation Measurements, 41, 1032-1039.

720 Murray, A. S., Wintle, A. G., 2000. Luminescence dating of quartz using an improved single-aliquot

721 regenerative-dose protocol. Radiation Measurements 32, 57-73.

722 Murray, A. S., Wintle, A. G., 2003. The single aliquot regenerative dose protocol: potential for

723 improvements in reliability. Radiation Measurements 37, 377-381.

724 Murray, A.S., Thomsen, K.J., Masuda, N., Buylaert, J.P., Jain, M., 2012. Identifying well-bleached quartz 725 using the different bleaching rates of quartz and feldspar luminescence signals. Radiation 726 Measurements 47, 688-695.

727 Nathan R. P., 2011. Numerical modelling of the environmental dose rate for trapped charge dating.

728 Unpublished PhD thesis, University of Oxford.

729 Nathan R.P, Mauz B., 2008. On the dose rate estimate of carbonate-rich sediments for trapped charge 730 dating. Radiation Measurements 43, 14-25.

731 Nathan, R.P., Thomas, P.J., Jain, M., Murray, A.S., Rhodes, E.J., 2003. Environmental dose rate 732 heterogeneity of beta radiation and its implications for luminescence dating: Monte Carlo modelling and 733 experimental validation. Radiation Measurements 37, 305-313.

734 Nielsen, A., Murray, A.S., Pejrup, M., Elberling, B., 2006. Optically stimulated luminescence dating of a 735 Holocene beach ridge plain in northern Jutland, Denmark. Quaternary Geochronology 1 (4), 305-312.

736 Olley, J.M., De Deckker, P., Roberts, R.G., Fifield, L.K., Yoshida, H., Hancock, G., 2004. Optical dating of deep-sea sediments using single grains of quartz: a comparison with radiocarbon. Sedimentary Geology $738169,175-189$.

739 Roberts, R.G., Galbraith, R.F., Yoshida, H., Laslett, G.M., Olley, J.M., 2000. Distinguishing dose 740 populations in sediment mixtures: a test of single-grain optical dating procedures using mixtures of 741 laboratory-dosed quartz. Radiation Measurements 32, 459-465.

742 Salvat F., Fernández-Varea, J.M. and Sempau, J., 2011. PENELOPE-2011: A Code System for Monte Carlo 743 Simulation of Electron and Photon Transport OECD NEA Data Bank/NSC DOC(2011)/5 (OECD Nuclear 744 Energy Agency, Issy-les-Moulineaux, 2011).

745 Salvat, F., Fernandez-Varea, J.M., Acosta, E., Sempau, J., 2011. PENELOPE - A Code System for Monte 746 Carlo Simulation of Electron and Photon Transport. Workshop Proceedings, Issy-les-Moulineaux, France, 747 (November 2001), AEN-NEA. 
Singhvi, A.K., Stokes, S.C., Chauhan, N., Nagar, Y.C., Jaiswal, M.K., 2011. Changes in natural OSL

749 sensitivity during single aliquot regeneration procedure and their implications for equivalent dose

750 determination. Geochronometria 38, 231-241.

751 Stokes, S., 1994a. The timing of OSL sensitivity changes in a natural quartz. Radiation Measurements 23, 752 601-605.

753 Stokes, S., 1994b. Optical Dating of selected late Quaternary aeolian sediments from the south western 754 United States. Unpublished D. Phil. thesis. Oxford University.

755 Thiel, C., Buylaert, J.P., Murray, A., Terhorst, B., Hofer, I., Tsukamoto, S., Frechen, M., 2011.

756 Luminescence dating of the Stratzing loess profile (Austria) - testing the potential of an elevated

757 temperature post-IR IRSL protocol. Quaternary International, 234, 23-31.

758 Thomsen, K.J., Murray, A.S., Bøtter-Jensen, L., 2005. Sources of variability in OSL dose measurements

759 using single grains of quartz. Radiation Measurements, 39, 47-61.

760 Thomsen, K.J., Murray, A.S., Bøtter-Jensen, L., Kinahan, J., 2007. Determination of burial dose in

761 incompletely bleached fluvial samples using single grains of quartz. Radiation Measurements 42 (3), 370-

762379.

763 Thomsen K. J., Murray A. S., Jain M., 2012. The dose dependency of the over-dispersion of quartz OSL

764 single grain dose distributions. Radiation Measurements 47, 732-739.

765 Tribolo, C., Mercier, N., Rasse, M., Soriano, S., Huysecom, E., 2010. Kobo 1 and L'Abri-aux-Vaches (Mali,

766 West Africa): two cases study for the optical dating of bio-turbated sediments. Quaternary

767 Geochronology 5, 317-323.

768 
770 Fig. 1: example of a Geant4 simulation of beta emission from potassium feldspars. The grains are

771

772

773

774

775

776

777

778

779

780

781

782

783

784

785

786

787

788

789

790

791

792

793

794

795

796

797

798

799

800

801

802 randomly packed using the LSD algorithm (Donev et al., 2005). Blue spheres represent potassium-rich feldspar grains, whereas grey ones represent quartz grains. Electron tracks generated inside feldspar grains are shown in red, while secondary photon tracks are shown in green.

Fig. 2: single grain OSL natural test dose response as a function of dose for (a) Ris $\varnothing$ calibration quartz (annealed by heating and then given a 4.81 Gy gamma dose), quartz from the intercomparison sample bleached in a daylight simulator (Hönle SOL 2) at a distance of $80 \mathrm{~cm}$ for 3 hours and then given gamma doses of $1.92 \mathrm{~Gy}(\mathrm{~b}), 4.81 \mathrm{~Gy}$ (c) and $9.62 \mathrm{~Gy}(\mathrm{~d})$, and natural (e).

Fig. 3: Q-Q plots of the standardised residuals from CAM $\mathrm{CL}_{\mathrm{L}}$ (left) and CAM (right). Ris $\varnothing$ calibration quartz (a); quartz from the intercomparison sample: bleached and given gamma doses of $1.92 \mathrm{~Gy}(\mathrm{~b}), 4.81 \mathrm{~Gy}$ (c) and 9.62 Gy (d).

Fig. 4: Geant4-simulated beta dose rate distribution from potassium-rich feldspar grains to single grains of quartz in the intercomparison sample.

Fig. 5: Q-Q plots of the standardised residuals from $C A M_{U L}$ (left) and CAM (right), for the natural $D_{e}$ distribution from the intercomparison sample.

Fig. 6: The different grain size distributions simulated with Geant4, corresponding to well-sorted sands. While the shape of the distributions is unchanged, the grain sizes are multiplied by different factors to investigate the effect of mean grain size on the single-grain dose rate distributions. The shaded bar indicates the 180-250 $\mu \mathrm{m}$ fraction, from which each grain is treated as an independent dosimeter.

Fig. 7: Examples of beta dose rate distributions from potassium feldspar in well-sorted sands for different potassium contents and mean grain sizes. The relative standard deviation is indicated in each case (RSD). Left: the potassium content is fixed (1.10\%) but the mean grain size increases from top to bottom. Right: the mean grain size is fixed $(360 \mu \mathrm{m})$ but the potassium content increases from top to bottom.

Fig. 8: Relative standard deviation, obtained with Geant4 simulations, of single grain beta dose rate distributions from potassium, as a function of potassium content for different grain sizes.

Fig. 9: Relative standard deviation, obtained with Geant4 simulations, of total dose rates to single grains of quartz, as a function of potassium content for different grain sizes. Total dose rate is $1 \mathrm{~Gy} . \mathrm{ka}^{-1}$ (a), 2 $\mathrm{Gy} \cdot \mathrm{ka}^{-1}$ (b), $3 \mathrm{~Gy} \cdot \mathrm{ka}^{-1}$ (c). The dashed lines indicate the range of most likely values for potassium content in each case (see text for details).

Fig. 10: Single grain OSL absolute (left) and relative (right) uncertainties as a function of dose. (a), (b): intercomparion sample, 4.81 Gy gamma distribution. (c), (d): intercomparison sample, natural distribution. Inset in (c): without the 3 high uncertainty points. 


\begin{tabular}{ccccccccc}
\hline $\mathrm{K}(\%)$ & $\mathrm{U}(\mathrm{ppm})$ & $\mathrm{Th}(\mathrm{ppm})$ & $\begin{array}{c}\text { Water } \\
\text { content } \\
(\%)\end{array}$ & $\begin{array}{c}\text { Gamma } \\
\text { dose-rate } \\
\left(\mathrm{Gy} \cdot \mathrm{ka}^{-1}\right)\end{array}$ & $\begin{array}{c}\text { Beta dose- } \\
\text { rate }\left(\mathrm{Gy} . \mathrm{ka}^{-1}\right)\end{array}$ & $\begin{array}{c}\text { Cosmic } \\
\left(\mathrm{Gy}_{\mathrm{k}} \mathrm{k}^{-1}\right)\end{array}$ & ${\left.\text { Total (Gy.ka }{ }^{-1}\right)}^{\text {Fraction }}$ & $\begin{array}{c}\text { contributed by } \\
\text { beta from K }\end{array}$ \\
\hline $1.06 \pm 0.02$ & $0.42 \pm 0.02$ & $1.38 \pm 0.04$ & 12 & $0.33 \pm 0.01$ & $0.74 \pm 0.03$ & 0.17 & $1.24 \pm 0.06$ & 0.50 \\
\hline
\end{tabular}

Table 1: Radiometric and dose-rate data for the inter-comparison sample, as measured in IRAMAT-CRP2A. 


\begin{tabular}{cc|cc|cc}
\multicolumn{2}{c|}{ Quartz OSL } & \multicolumn{2}{c|}{ Feldspar IRSL at $50{ }^{\circ} \mathrm{C}$} & \multicolumn{2}{c}{ Feldspar postIR-IRSL at $290{ }^{\circ} \mathrm{C}$} \\
\hline $\mathrm{D}_{\mathrm{e}}(\mathrm{Gy})$ & Age $(\mathrm{ka})$ & $\mathrm{D}_{\mathrm{e}}(\mathrm{Gy})$ & Age $(\mathrm{ka})$ & $\mathrm{D}_{\mathrm{e}}(\mathrm{Gy})$ & Age $(\mathrm{ka})$ \\
\hline $4.73 \pm 0.23$ & $3.81 \pm 0.26$ & $6.90 \pm 0.30$ & $4.28 \pm 0.27$ & $13.7 \pm 0.6$ & $6.69 \pm 0.36$
\end{tabular}

Table 2: Ages obtained for the intercomparison sample, using quartz OSL, feldspar IRSL at $50^{\circ} \mathrm{C}$ and post-IR IRSL at $290^{\circ} \mathrm{C}$.

${ }^{a}$ the Huntley and Lamothe fading correction model was applied. 


\begin{tabular}{|c|c|c|c|c|c|c|c|c|c|c|c|c|}
\hline & $\begin{array}{l}\text { Given dose } \\
\text { (Gy) }\end{array}$ & $n^{b}$ & $\begin{array}{l}\text { Avg. } \\
\text { error } \\
(\%)^{c}\end{array}$ & De (Gy) & CAM & $\begin{array}{c}\text { Recovery } \\
\text { ratio }\end{array}$ & De (Gy) & OD (Gy) & $\begin{array}{c}\text { OD (\% of } \\
\left.D_{e}\right)\end{array}$ & $\begin{array}{c}\text { Recovery } \\
\text { ratio }\end{array}$ & Unweigh & $\begin{array}{l}\text { Rerage } \\
\text { Recovery } \\
\text { ratio }\end{array}$ \\
\hline \multirow[t]{4}{*}{ InterComp. } & $1.92 \pm 0.03$ & 85 & 27 & $1.86 \pm 0.06$ & $17 \pm 3$ & $0.97 \pm 0.03$ & $1.79 \pm 0.07$ & $0.35 \pm 0.05$ & 0.20 & $0.93 \pm 0.03$ & $1.88 \pm 0.08$ & $0.98 \pm 0.04$ \\
\hline & $4.81 \pm 0.07$ & 71 & 21 & $4.74 \pm 0.16$ & $15 \pm 3$ & $0.98 \pm 0.03$ & $4.58 \pm 0.17$ & $0.88 \pm 0.12$ & 0.19 & $0.95 \pm 0.03$ & $4.63 \pm 0.12$ & $0.96 \pm 0.02$ \\
\hline & $9.62 \pm 0.14$ & 108 & 13 & $9.63 \pm 0.28$ & $15 \pm 2$ & $1.00 \pm 0.02$ & $9.49 \pm 0.28$ & $1.5 \pm 0.2$ & 0.16 & $0.99 \pm 0.02$ & $9.81 \pm 0.33$ & $1.02 \pm 0.03$ \\
\hline & Nat & 123 & 23 & $4.51 \pm 0.15$ & $23 \pm 2$ & & $4.38 \pm 0.16$ & $1.2 \pm 0.1$ & 0.28 & & $4.56 \pm 0.19$ & \\
\hline 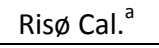 & $4.81 \pm 0.07$ & 369 & 13 & $108 \pm 1$ & $15 \pm 1$ & & $106 \pm 1$ & $18 \pm 1$ & 0.17 & & $108 \pm 1$ & \\
\hline
\end{tabular}

Table 3: Statistics of the different single-grain quartz $D_{e}$ distributions.

${ }^{a}$ for the Ris $\varnothing$ calibration quartz, all measured equivalent doses are given in seconds rather than in Gy, as the result is used for dose-rate calibration of the reader.

${ }^{\mathrm{b}}$ Number of accepted grains.

${ }^{c}$ Average relative uncertainty on individual dose estimates. 


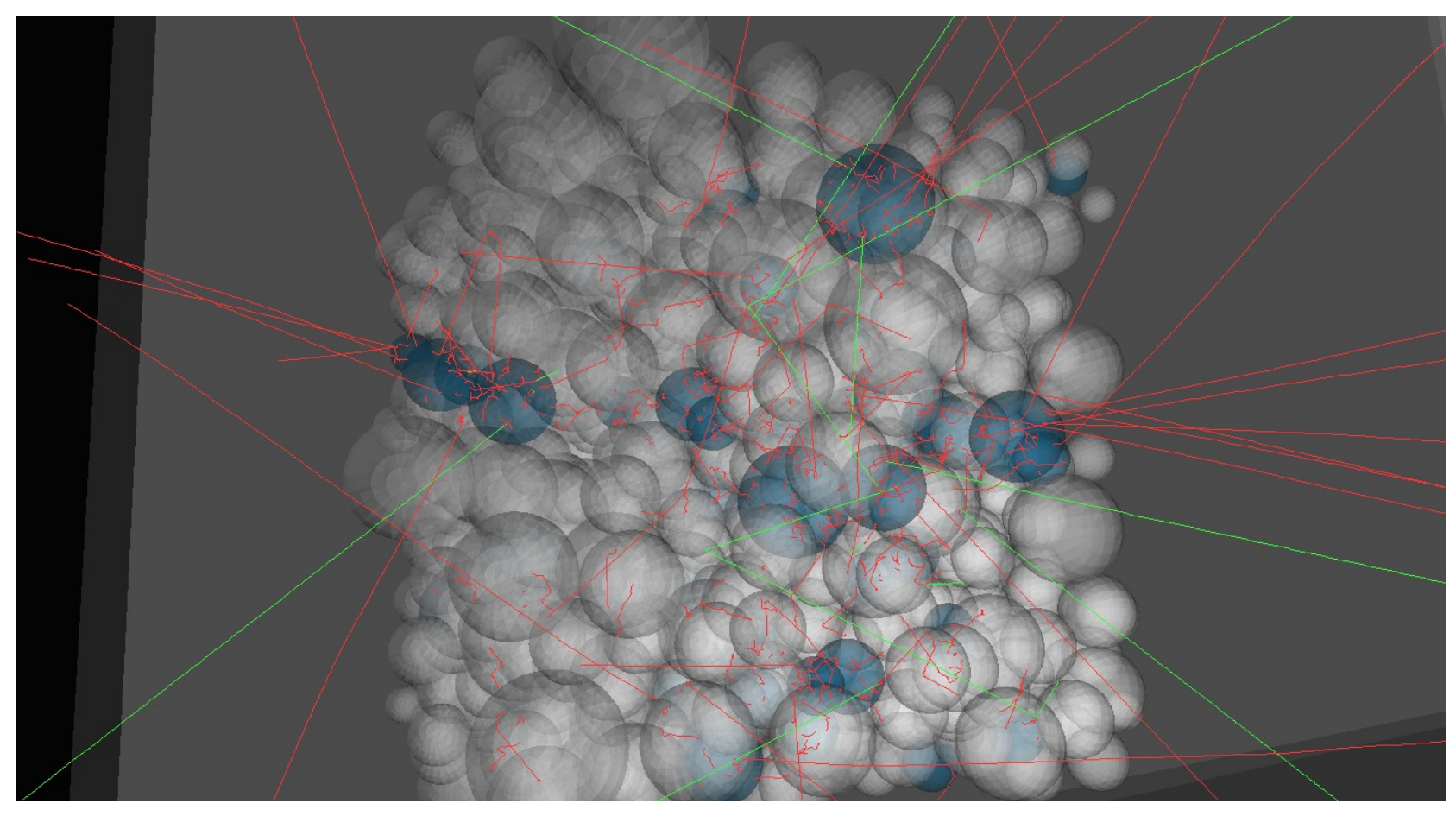

Fig. 1 

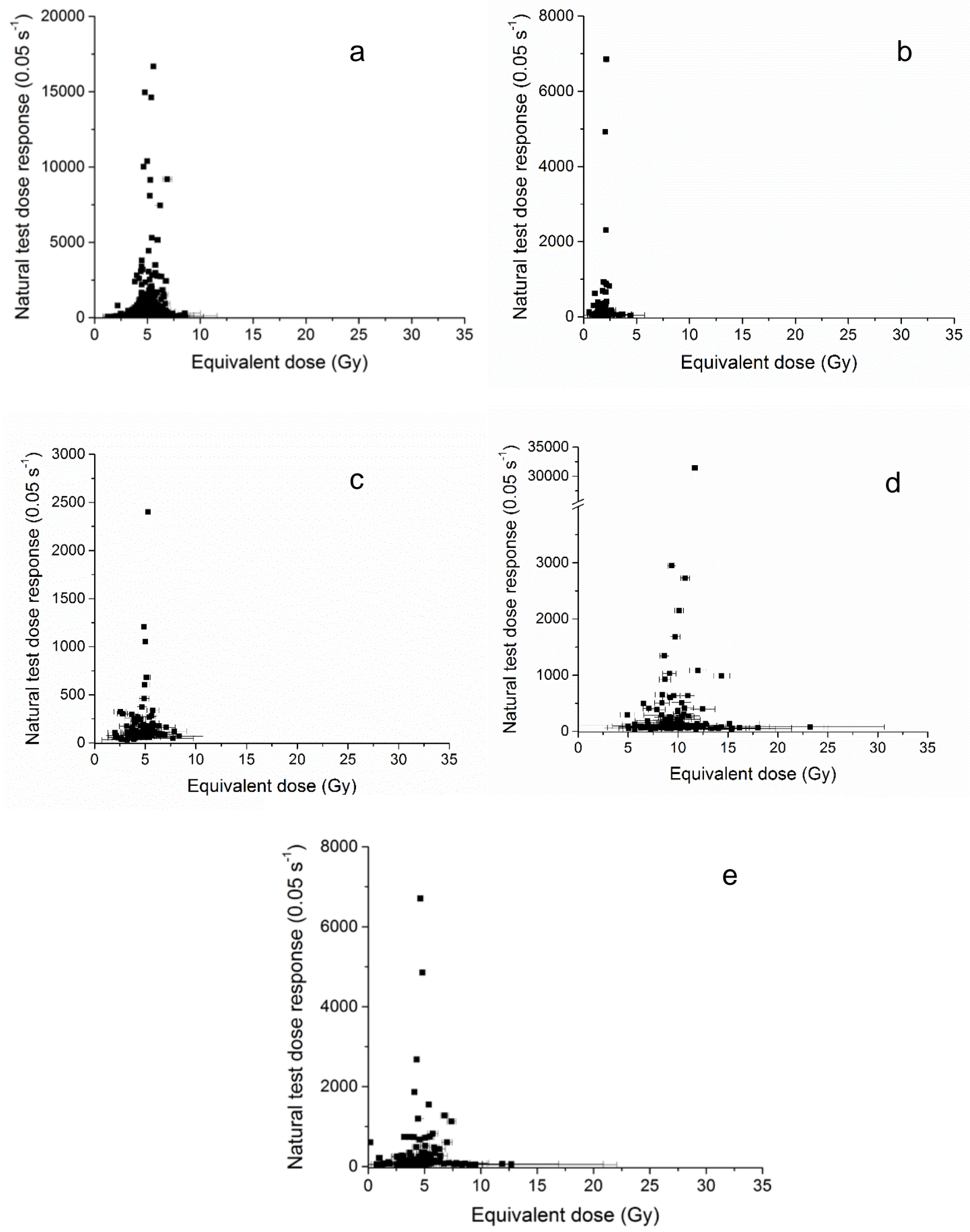

Fig. 2: 


$$
\begin{aligned}
& 1<1< \\
& 1<1<
\end{aligned}
$$




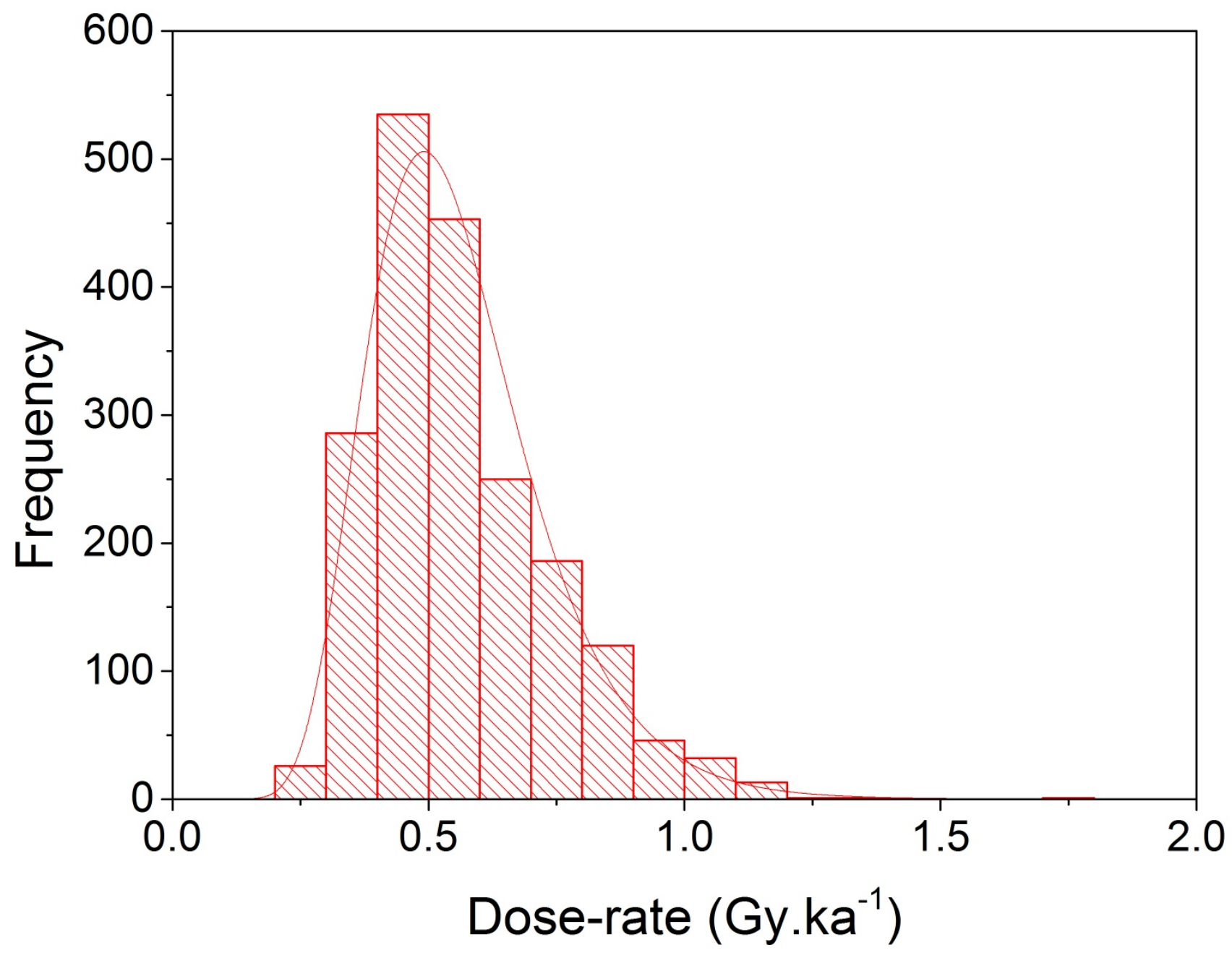

Fig. 4: 

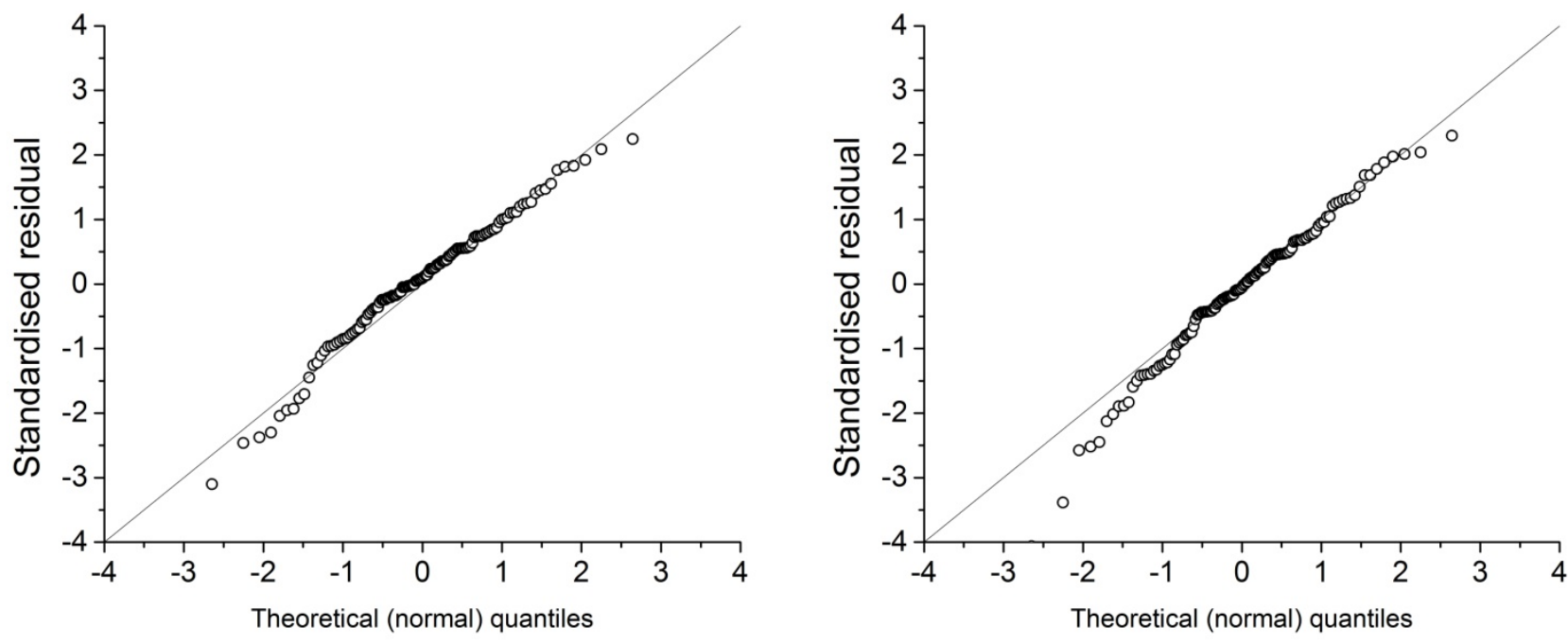

Fig. 5: 


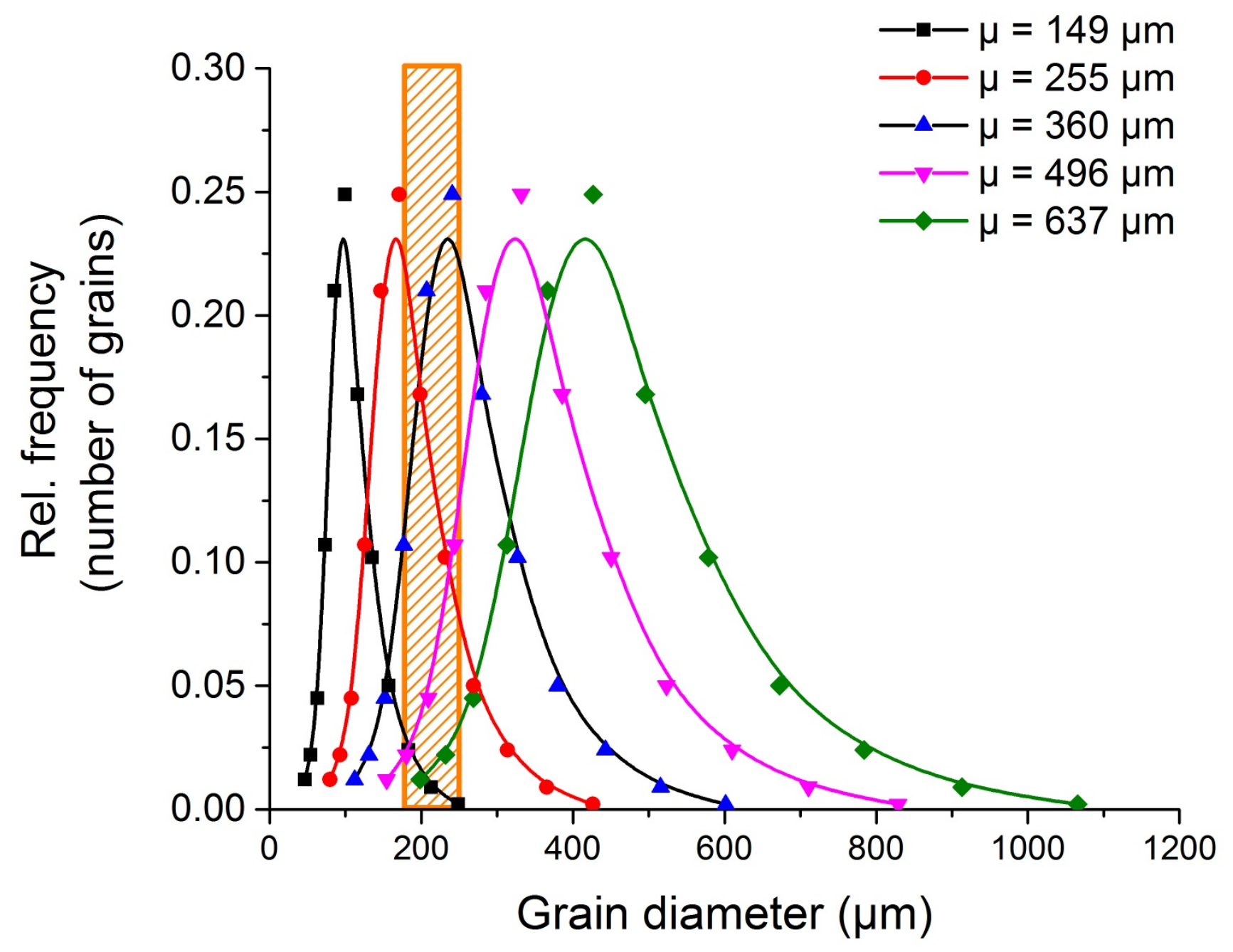

Fig. 6: 

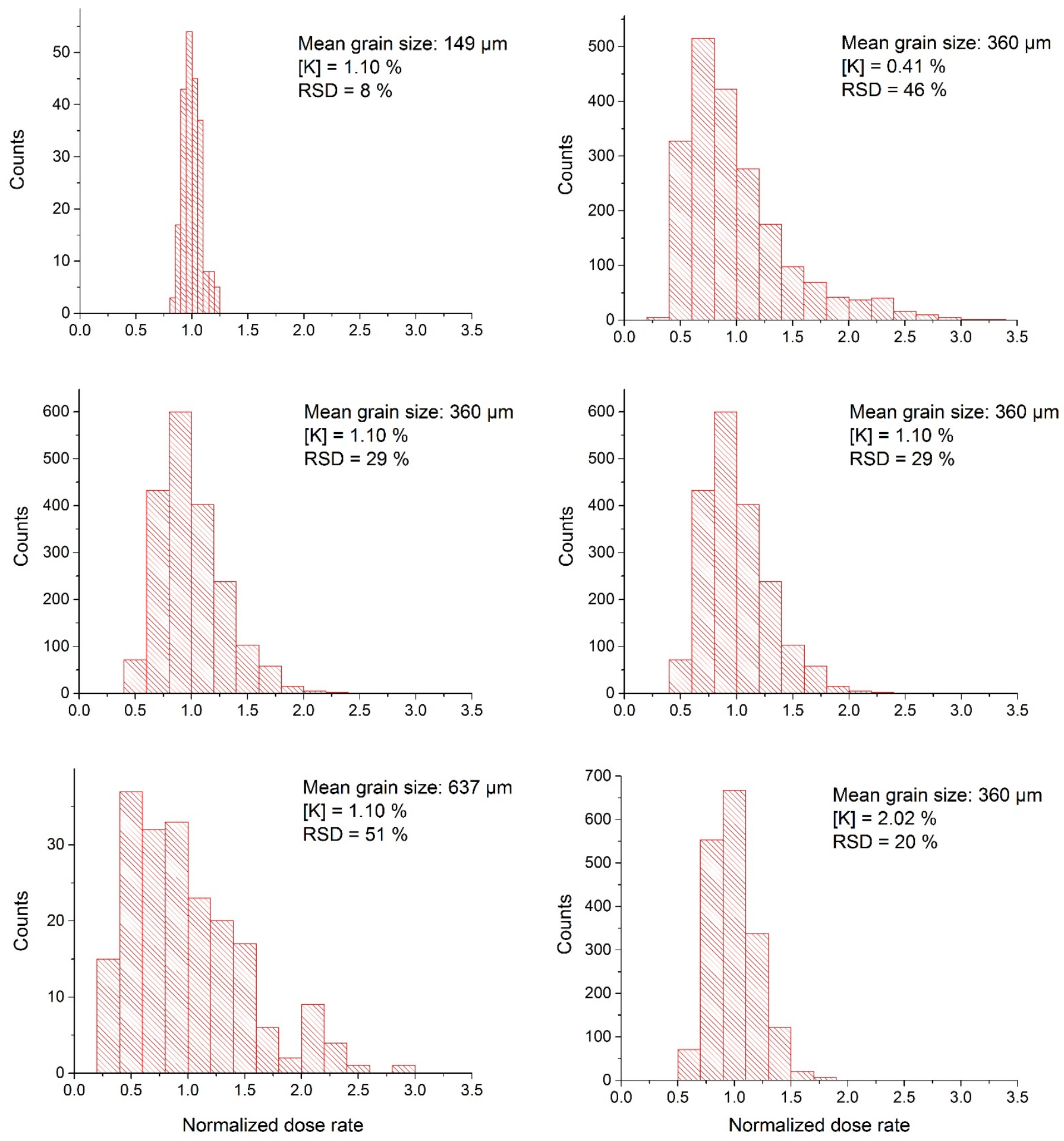

Fig. 7 


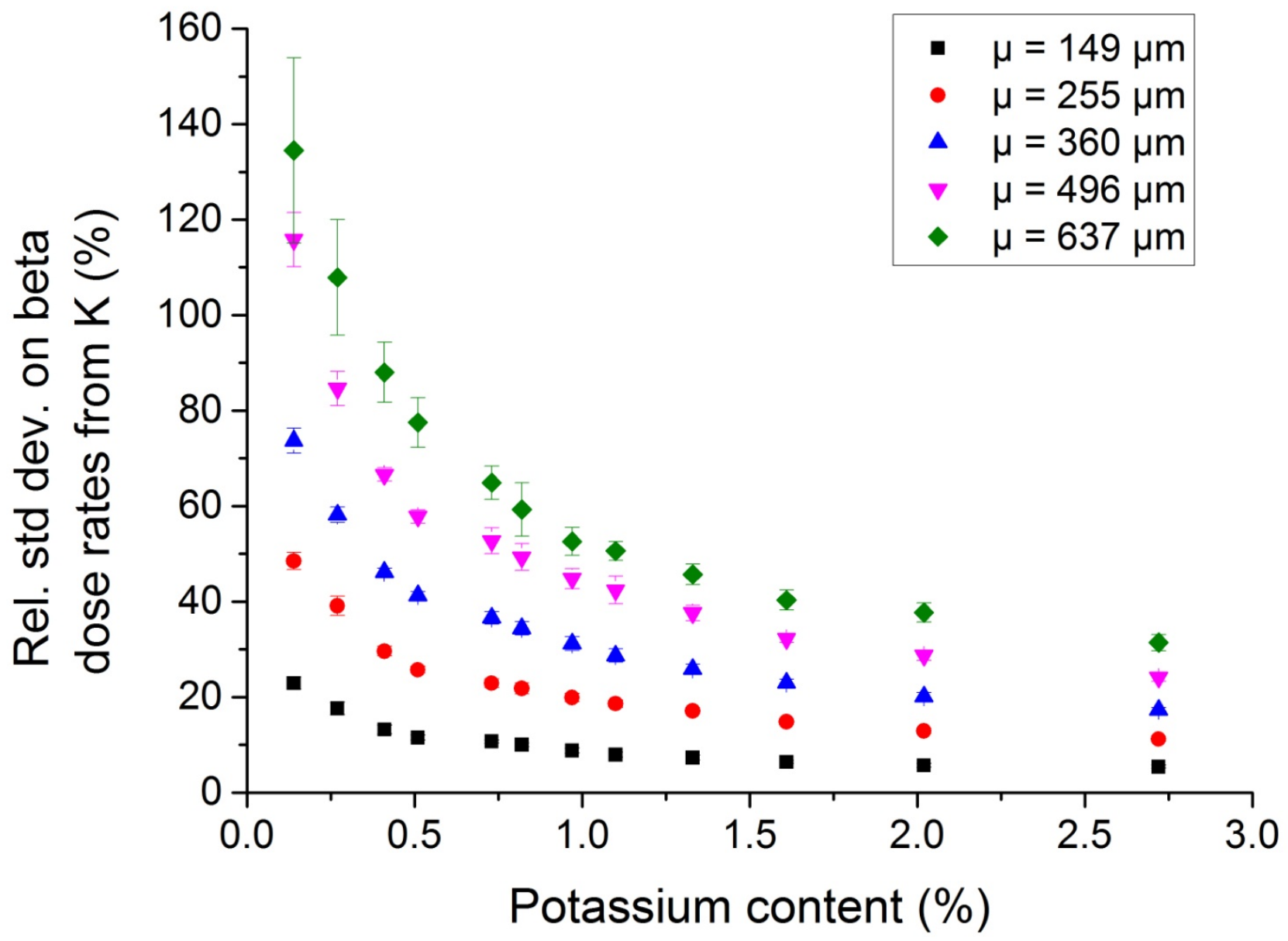

Fig. 8 
a

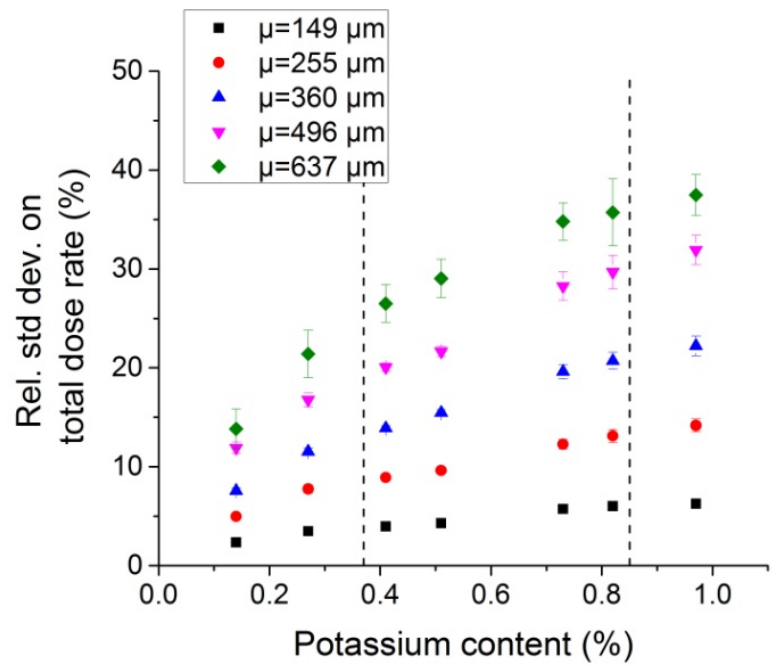

b

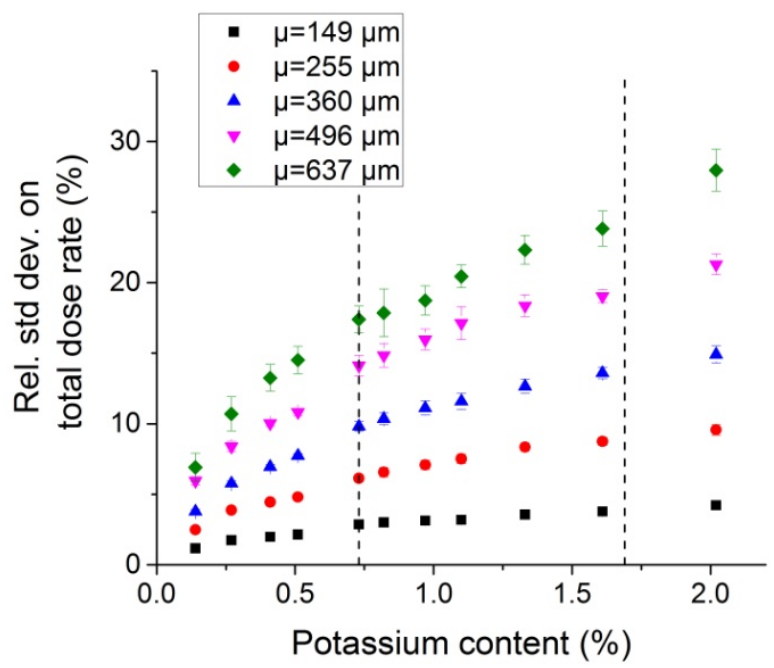

C

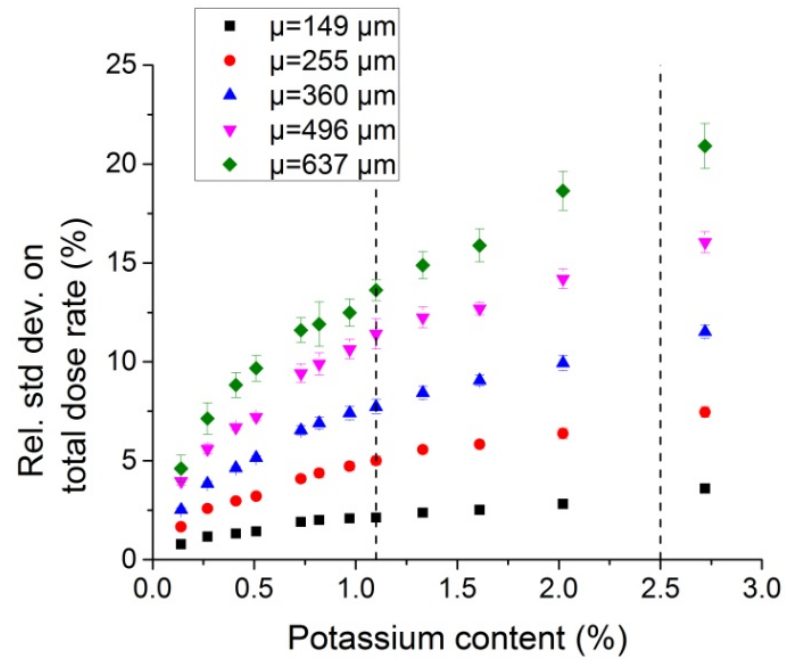

Fig. 9 
a

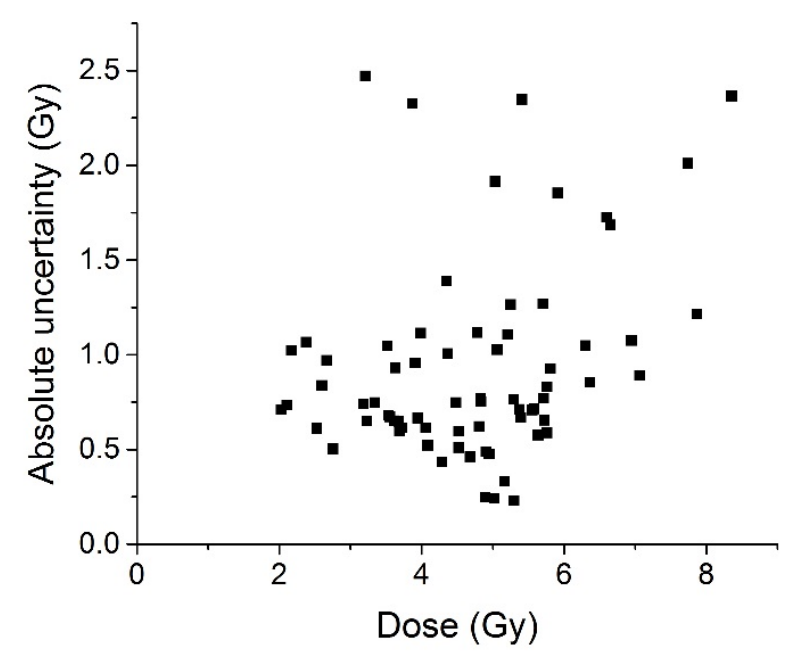

C

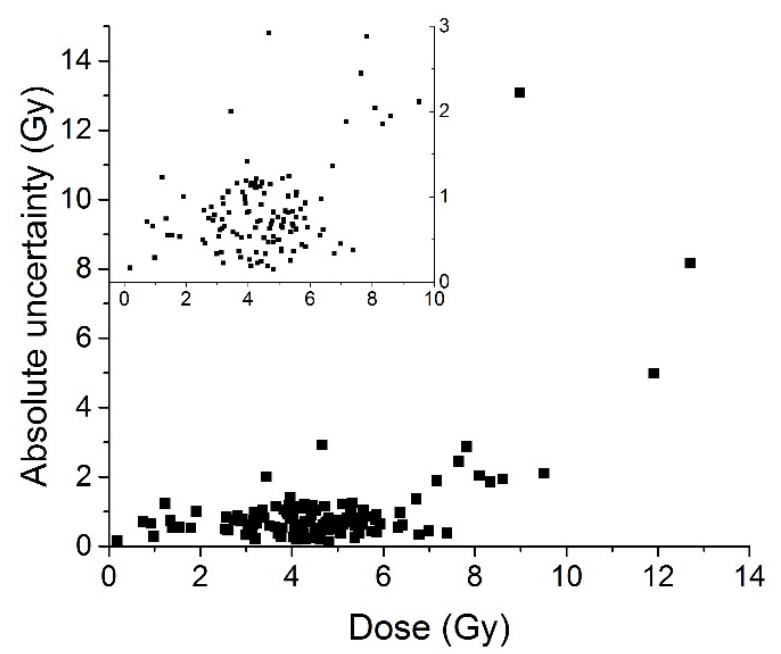

b

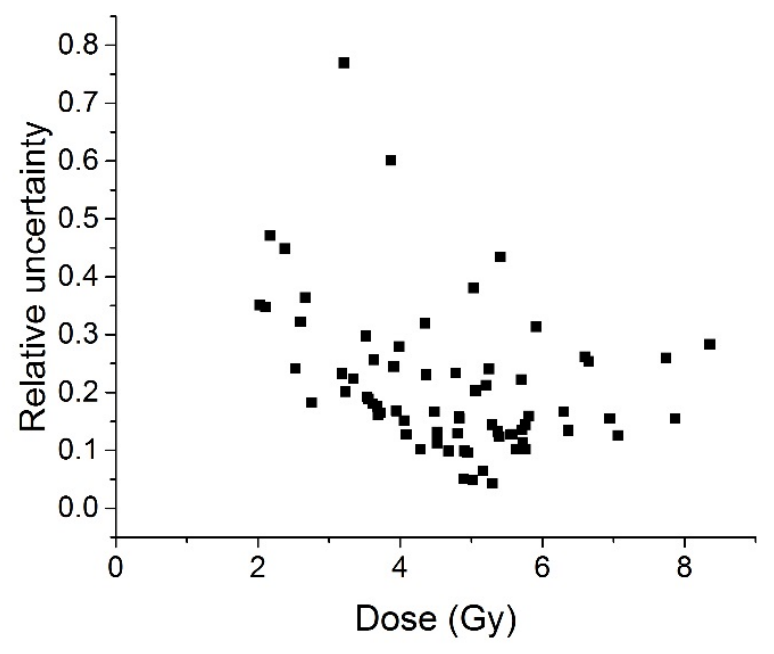

d

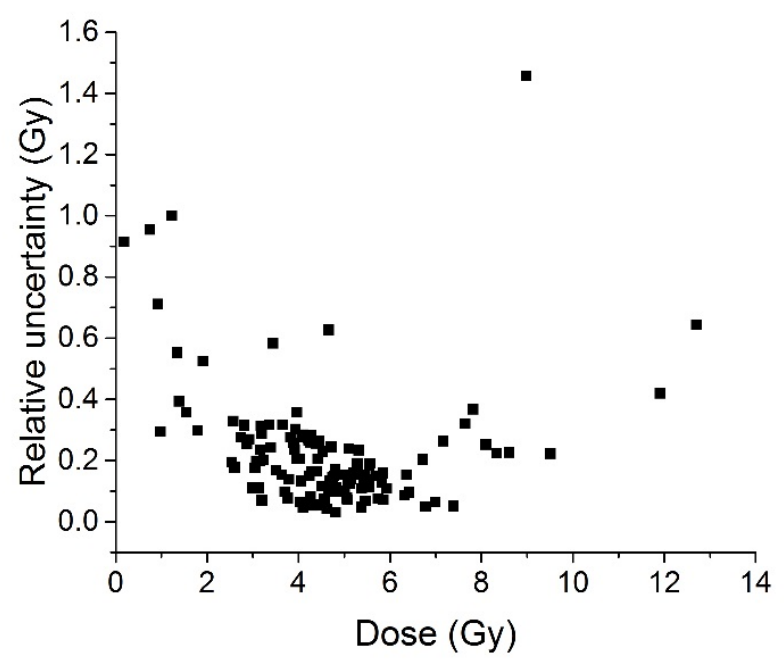

Fig. 10 